\title{
A super-resolution analysis of the DSHARP survey: Substructure is common in the inner 30 au
}

\author{
Jeff Jennings, ${ }^{1 \star}$ Richard A. Booth, ${ }^{2}$ Marco Tazzari, ${ }^{1}$ Cathie J. Clarke, ${ }^{1}$ and \\ Giovanni P. Rosotti ${ }^{3,4}$ \\ ${ }^{1}$ Institute of Astronomy, University of Cambridge, Madingley Road, Cambridge CB3 OHA, UK \\ ${ }^{2}$ Astrophysics Group, Imperial College London, Prince Consort Road, London SW7 2AZ, UK \\ ${ }^{3}$ Leiden Observatory, University of Leiden, P.O. Box 9500, Leiden NL-2300 RA, Netherlands \\ ${ }^{4}$ School of Physics and Astronomy, University of Leicester, Leicester LE1 7RH, UK
}

Accepted XXX. Received YYY; in original form ZZZ

\begin{abstract}
The DSHARP survey evidenced the ubiquity of substructure in the $\mathrm{mm}$ dust distribution of large, bright protoplanetary discs. Intriguingly, these datasets have yet higher resolution information that is not recovered in a CLEAN image. We first show that the intrinsic performance of the CLEAN algorithm is resolution-limited. Then analyzing all 20 DSHARP sources using the 1D, super-resolution code Frankenstein (frank), we accurately fit the 1D visibilities to a mean factor of 4.3 longer baseline than the Fourier transform of the CLEAN images and a factor of 3.0 longer baseline than the transform of the CLEAN component models. This yields a higher resolution brightness profile for each source, identifying new substructure interior to 30 au in multiple discs; resolving known gaps to be deeper, wider, and more structured; and known rings to be narrower and brighter. Across the survey, high contrast gaps are an average $14 \%$ wider and $44 \%$ deeper in the frank profiles relative to CLEAN, and high contrast rings are an average $26 \%$ narrower. Categorizing the frank brightness profiles into trends, we find that the relative scarcity of features interior to 30 au in the survey's CLEAN images is an artifact of resolving power, rather than an intrinsic rarity of inner disc (or compact disc) substructure. Finally the rings in the frank profiles are narrower than the previously inferred deconvolved widths, indicating smaller $\alpha /$ St ratios in the local gas disc.
\end{abstract}

Key words: techniques: interferometric, submillimetre: general, submillimetre: planetary systems, protoplanetary discs, planets and satellites: detection, methods: data analysis

\section{PHYSICAL AND METHODOLOGICAL CONTEXTS}

Interferometric observations of the dust and gas components of protoplanetary discs provide the highest resolution information available on the structure of these sources, which in turn traces the planetary companions and physical mechanisms responsible. At the highest angular resolutions achieved to date in the sub-mm $-\mathrm{mm}$ with the Atacama Large Millimeter Array (ALMA; beam widths of $\approx 25-75$ mas corresponding to $\approx 1-10 \mathrm{au})^{1}$, studies prior to DSHARP first identified, characterized and analyzed an abundance of dust substructure in individual systems (ALMA Partnership et al. 2015; Andrews et al. 2016; Clarke et al. 2018). The DSHARP survey (Andrews et al. 2018; Huang et al. 2018a) then confirmed that annular gaps and rings are ubiquitous in the dust of large, bright discs around single stars. The survey additionally found a nonnegligible occurrence rate of nonaxisymmetric dust substructure in the

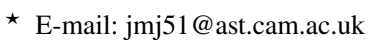

1 Notation: we use $\approx$ to mean 'approximately equal to' and $\sim$ to mean 'of order.'
}

form of spirals arms (Huang et al. 2018b; Kurtovic et al. 2018) and azimuthally localized brightness arcs (Isella et al. 2018; Pérez et al. 2018). Studies since DSHARP for individual systems at comparable resolutions have upheld the high occurrence rate of dust substructure (e.g., Kudo et al. 2018; Keppler et al. 2019; Pinte et al. 2019; Pérez et al. 2019).

Analyses of interferometric datasets in the protoplanetary disc community, including in the DSHARP survey, generally rely on images generated with the CLEAN deconvolution technique (Högbom 1974; Clark 1980; Cornwell 2008). In the reconstruction of a model image from an interferometric measurement, a fundamental challenge is accounting for unsampled spatial frequencies (baselines). A direct Fourier transform of the visibilities at sampled baselines (i.e., an assumption of zero power on unsampled baselines) yields a 'dirty image,' i.e., the sky brightness convolved with the instrument's point spread function (PSF; 'dirty beam'). This convolution introduces artifacts into the dirty image due to the PSF's sidelobe structure, and the CLEAN algorithm is a nonlinear, procedural approach to remove these artifacts (deconvolution). To do this, CLEAN 
begins with a 'residual image' that is equal to the dirty image, then iteratively: finds the peak flux in the residual image, adds a corresponding 'component' (in the simplest case, a Dirac $\delta$ function) to the 'CLEAN model' (an image composed only of the CLEAN components), and subtracts this component convolved with the dirty beam from the residual image. At the end of this iteration, the CLEAN model is convolved with the 'CLEAN beam' (a Gaussian fit to the primary lobe of the dirty beam), and the final state of the residual image is added to this to form the 'CLEAN image' commonly presented as the astronomical observation.

While the CLEAN algorithm is the standard and highly successful technique used across much of radio interferometry, the procedure imposes artificial resolution loss in the image reconstruction, primarily from convolution of the CLEAN model with the CLEAN beam. This causes all features in the CLEAN image, regardless of their scale, to be smeared in resolution over the size of the beam. For the 1D (radial) brightness profile of a source, convolution induces a reduction in amplitude of all disc features, an overestimate of ring widths, and an underestimate of gap widths.

As we will demonstrate, 'super-resolution' imaging techniques can overcome the resolution limits of the CLEAN algorithm. ${ }^{2}$ These methods thus have the capacity to provide new insights into a source's substructure from existing datasets, better informing physical inference and follow-up observing strategies. While superresolution approaches have been applied to individual DSHARP discs, namely parametric visibility fitting in Guzmán et al. 2018a, Isella et al. 2018 and Pérez et al. 2018, no study has yet examined the entire DSHARP sample.

Super-resolution fitting techniques used in (and in some cases tailored to) the protoplanetary disc field can be divided into image plane and Fourier domain approaches. Image plane procedures include the maximum entropy method (Gull \& Daniell 1978; Narayan \& Nityananda 1986; Sutton \& Wandelt 2006; Casassus et al. 2006, 2013; Chael et al. 2016) and sparse modeling (Honma et al. 2014; Akiyama et al. 2017; Kuramochi et al. 2018; Nakazato et al. 2019), with the broad class of regularized maximum likelihood techniques being actively used in Very Long Baseline Interferometry (Event Horizon Telescope Collaboration et al. 2019, and references therein) and for application to protoplanetary discs (Czekala et al. 2021). Fourier domain approaches include fitting the visibilities parametrically (Perkins et al. 2015; Tazzari et al. 2018) and nonparametrically (Jennings et al. 2020).

In this work we characterize substructure at super-resolution scales in all 20 DSHARP discs using the 1D code frank (Jennings et al. 2020), which reconstructs a disc's brightness profile by nonparametrically fitting the azimuthally averaged visibility distribution. ${ }^{3}$ Sec. 2 summarizes the frank modeling approach and its limitations. Sec. 3 then examines the resolution limitations of CLEAN images and models in real and visibility space (Sec. 3.1), compares the accuracy of brightness profiles extracted from the CLEAN images and models with the frank visibility fits for the DSHARP sources (Sec. 3.2), and summarizes the principles of comparing frank to CLEAN (Sec. 3.3). In Sec. 4 we present the super-resolution frank fits for each DSHARP source, then group the frank brightness profiles by previously unidentified substructure trends in Sec. 5 . We

2 By 'super-resolution' we mean an achieved fit resolution higher than the achieved CLEAN resolution, which we will quantify as distinct from the CLEAN beam width.

3 The code is available at https://github.com/discsim/frank. All frank fits in this work are available at https://zenodo.org/record/5587841. further use the super-resolution fits to identify a geometric viewing effect that can imprint on disc images. Sec. 6 summarizes our findings and briefly places them in the context of super-resolution substructure that may be present in other protoplanetary disc datasets, as well as the physical inference this can inform.

\section{MODEL}

For a full description of the model framework in frank, see Jennings et al. (2020). Here we briefly and qualitatively summarize the approach. frank reconstructs the azimuthally averaged brightness profile of a source as a function of disc radius by directly fitting the real component of the deprojected, unbinned visibilities as a function of baseline. ${ }^{4}$ The brightness profile is determined nonparametrically by fitting the visibilities with a Fourier-Bessel series, which is linked to the real space profile by a discrete Hankel transform (Baddour \& Chouinard 2015). The Fourier transform of a circle has a Bessel function kernel, making the discrete Hankel transform (DHT) a natural basis for circular (at least to zeroth order) protoplanetary discs. A Gaussian process regularizes the fit, with the covariance matrix nonparametrically learned from the visibilities under the assumption that this matrix is diagonal in Fourier space. The free parameters (diagonal elements) of the matrix correspond to the power spectrum of the reconstructed brightness profile. The approach is largely built on that in Oppermann et al. (2013).

The model has five free parameters; variation in reasonable choices for three of these (the outer radius and number of points used in the fit, and the floor value for the power spectral mode amplitudes) has a trivial effect on the recovered profile. Of the remaining two, $\alpha$ sets the signal-to-noise (SNR) threshold at which the model stops fitting the data, with a larger $\alpha$ resulting in a higher SNR threshold. The choice of $\alpha$ effectively corresponds to a maximum baseline beyond which the model does not attempt to fit the visibilities. This is relevant for the DSHARP datasets, as they all become noisedominated typically at $\gtrsim 5 \mathrm{M} \lambda$, while the maximum baseline is $\approx 10 \mathrm{M} \lambda$. In practice we manually choose an $\alpha$ value to fit out to the baseline at which the binned visibility SNR begins to oscillate about $S N R=1$ (due to the $u v$ sampling becoming highly sparse). The SNR is assessed with $20 \mathrm{k} \lambda$ bins of the real component of the visibilities, using SNR $=\mu^{2} / \sigma^{2}$, where $\mu$ is the mean visibility amplitude in each bin and $\sigma$ the standard deviation. Pushing the fit out to these long baselines always comes at the cost of fitting some noise, which imprints on the brightness profile as rapid oscillations, usually with very low amplitude (typically $<1 \%$ of the profile's peak brightness; as an example, see the fit residuals in Fig. 8 of Jennings et al. 2020). To suppress these noisy oscillations, the remaining free parameter $w_{\text {smooth }}$ varies the spatial frequency scale over which the visibility SNR is averaged when building the power spectrum. A nonzero $w_{\text {smooth }}$ prevents regions of artificially steep gradient in the power spectrum that are due to undersampled baselines.

For the DSHARP datasets, we use $\alpha$ and $w_{\text {smooth }}$ values within the ranges $1.01 \leq \alpha \leq 1.50$ and $10^{-4} \leq w_{\text {smooth }} \leq 10^{-1}$, tailoring choices to the unique visibility distribution and noise properties of

4 We will use the disc geometries and phase centers in Huang et al. (2018a) to deproject the DSHARP datasets. Those values were determined in the image plane by either fitting ellipses to individual annular rings or fitting a 2D Gaussian to the image. Across all datasets, we have tested both fitting a 2D Gaussian to the visibilities and fitting the visibilities nonparametrically to determine the geometry and phase center. In general we have found close agreement with the published values and so default to those. 
each dataset. We favor the smaller values within these ranges in order to reduce the constraint placed by the Gaussian process prior on the brightness profile reconstruction.

To fit each dataset, we download the self-calibrated and multi-configuration combined continuum measurement sets from https://bulk.cv.nrao.edu/almadata/lp/DSHARP. Before extracting the visibilities using the export_uvtable function of the uvplot package (Tazzari 2017), we apply channel averaging (to obtain 1 channel per spectral window) and time averaging (30 sec) to all spectral windows in the original MS table. The frank fit takes $\lesssim 1$ min for each resulting visibility distribution.

To generate images of the frank residual visibilities in this work, we produce measurement sets from the frank residual UV tables, then use the tclean scripts from the DSHARP website to image. These scripts yield CLEAN beams that are often larger than those in the fits files on the website, though only by $1-2$ mas along either axis. The only exception is HD 143006, where the CLEAN beam is $36 \times 53$ mas in the . fits file, while the tclean script yields $47 \times 48$ mas (this may be due to slightly different versions of CASA used). For consistency with the imaged frank residuals, we will therefore show CLEAN images generated by applying the published tclean scripts to the published measurement sets, rather than showing the published . fits images.

\subsection{Point source-corrected fits}

Eleven of the 20 DSHARP datasets do not clearly converge on zero visibility amplitude at their longest baselines, exhibiting a mean value of $0<\operatorname{Re}(V)<1 \mathrm{mJy}$ (relative to a peak visibility amplitude of $\approx 100 \mathrm{mJy}$ ). This seems to indicate that the observations are detecting a point-like source - namely the innermost disc, whose brightness increases sharply toward $r=0$. A frank visibility fit strongly drives to zero once its SNR threshold is reached (which is a deliberate choice motivated by the high uncertainty in extrapolating the fit beyond the longest well-sampled baselines). And a steep slope in the fit at any baseline is represented in the brightness profile as structure on the corresponding spatial scale. Thus for a dataset that does not converge on zero at long baselines, a steep slope in the frank fit prior to the baseline at which the visibilities converge on zero can impose false oscillations on the brightness profile. These oscillations manifest as a sinc-like function, at constant spatial period (the inverse of the spatial frequency location of the slope in Fourier space) and at an amplitude that diminishes away from $r=0$.

To prevent this artifacting, we have developed an extension to frank for a 'point source-corrected model' to effectively subtract a point source from the visibilities and fit the resulting 'residuals', which are centered on $\operatorname{Re}(V)=0$ at long baseline. By doing this we have implicitly assumed that there is a strong point source at the center of the disc. This model is one of an infinite number of choices to extrapolate the fitted visibility distribution to inaccessible scales (a requirement of any imaging algorithm) while remaining consistent with the observed data. The choice is however sensible, as it is both physically and practically motivated. Discs are expected to rapidly increase in brightness towards the star, and applying no point-source correction can lead to spurious, coherent oscillations in the recovered brightness profile.

A pure point source (Delta function) in real space transforms to a constant visibility amplitude at all baselines. While the innermost disc is not physically a Delta function, we find this approximation works well in an unresolved component fit. In the point source-corrected model, we first subtract a constant amplitude from the visibilities, equal to the mean offset from zero at the dataset's longest baselines (specifically, those beyond the point at which the binned visibility SNR begins to oscillate about $S N R=1$ ). Then we perform a standard frank fit on the 'residual' visibilities, and finally add the constant amplitude offset back into the frank visibility fit. Empirically, we have found this approach does a reasonable job of preventing artifacting in the frank brightness profile for each of the 11 DSHARP datasets whose visibilities do not clearly converge on zero (we will note these discs in Sec. 3). However the technique does not fully suppress oscillations in the brightness profile in some sources, particularly in the innermost disc. In these cases the amplitude and spatial period of oscillations is sensitive to the point source amplitude; an example is shown in Sec. A. We therefore assess the associated uncertainty by comparing, for each source, the fit that uses the point source amplitude as determined above with a fit that uses a $1.5 \times$ larger point source amplitude (an example case is discussed in Sec. A). This is motivated by a model with a larger point source amplitude effectively fitting the data to shorter baseline, which yields a more conservative estimate of small scale substructure in the brightness profile. In the main text we show the difference between the profiles of these two point source fits as an informal uncertainty band.

\subsection{Model limitations}

The model's notable limitations in the context of this analysis are:

(i) The 1D (axisymmetric) approach fits for the azimuthal average of the visibility data at each baseline. The model is thus inaccurate for any annulus at which the brightness is not perfectly symmetric, averaging an asymmetry over $2 \pi$ in azimuth. Azimuthally localized features such as a bright arc then appear in the 1D brightness profile as a plateau or 'bump' (depending on their relative brightness; we will identify specific instances). Especially for superresolution features not seen in a CLEAN image, it can be difficult in some cases to distinguish the artifact of an asymmetry from an underresolved annular feature using only the 1D frank brightness profile and observed visibilities.

To partially resolve this ambiguity, we image the frank fit residual visibilities to exploit that the axisymmetric model fits for the average brightness at each annulus. This effectively isolates azimuthal asymmetries in the imaged residuals ${ }^{5}$, allowing us to identify radii at which asymmetries are coincident with features in the reconstructed brightness profile. But for discs that have overlapping annular structures and azimuthal asymmetries (in DSHARP, discs with prominent spirals), interpretation is more ambiguous. We generate a frank residual image using the same imaging parameters as the CLEAN image of the source; the residual image is thus convolved and at lower resolution than the frank brightness profile. Assessment of these residual images is therefore not a substitute for analysis with a 2D super-resolution model.

The axisymmetric approach in frank is also incorrect for fields of view with multiple sources (AS 205 and HT Lup in the DSHARP sample), as these are asymmetric on large scales. Structure on the scale of a secondary disc must at some level bias the frank fit for the primary, and we have tested the severity of this effect by refitting the

5 While azimuthal asymmetries are 'isolated' in the imaged frank residuals, their brightness in the image is biased because the 1D fit cannot localize flux azimuthally. The fit recovers the total flux in any annulus correctly. But a feature such as a bright arc that is localized in azimuth will have its imaged brightness biased low, because the fit distributes it over the full $2 \pi$ in azimuth. 
HT Lup dataset after subtracting out the secondary disc seen in the CLEAN image. We found this to only weakly alter the morphology of the frank brightness profile for HT Lup. We verified this weak sensitivity with mock datasets containing brightness asymmetries, in which we found a frank brightness profile to be trivially altered by structure on a given scale at radii where that structure is not present. Regardless, application of the model to a field of view with multiple sources is formally incorrect.

(ii) While frank produces an estimate of the uncertainty on the fitted brightness profile, the estimate is not reliable because reconstructing the brightness from Fourier data is an ill-posed problem (see the discussion of this in Jennings et al. 2020). In particular, we do not have a robust approach for accurately extrapolating visibility amplitudes in a given dataset beyond the longest baseline that frank fits. The uncertainty on the brightness profile produced by the model is an underestimate, and we thus do not show a formal uncertainty on any profile in this work (the uncertainty described in Sec. 2.1 is informal). The uncertainty on spatial scales well resolved by a frank fit is very low as demonstrated with mock data in Jennings et al. (2020). We note that the $1 \sigma$ contour typically shown as an uncertainty on CLEAN brightness profiles is also often an underestimate, as will be evident by comparing the CLEAN and frank profiles in this work. A valuable test of systematics in the extrapolation of any model is perhaps best achieved in practice by comparing observations of the same source at different resolutions (see, e.g., Yamaguchi et al. 2020 for this comparison using sparse modeling, or Jennings et al. 2020 for such a comparison with frank fits to moderate resolution and DSHARP observations of AS 209).

(iii) The current frank model fits for the brightness in linear space and is not positive definite (see Appendix C in Jennings et al. 2020). Consequently the frank brightness profile for a disc with a deep gap or an inner cavity can exhibit negative brightness in this region. We will enforce that such fits must have nonnegative brightness (which trivially affects the visibility domain fit) and will note discs for which we impose this constraint.

\section{METHODOLOGIES - ASSESSING EFFECTIVE CLEAN RESOLUTION AND FIT ACCURACY}

Here we motivate resolution limitations that affect CLEAN images and CLEAN models (Sec. 3.1), then compare the accuracy of brightness profiles extracted from CLEAN images and CLEAN models to the frank fits for all DSHARP datasets, quantifying the resolution improvement in frank (Sec. 3.2). We summarize the principles of comparing frank fits to CLEAN in Sec. 3.3.

\subsection{CLEAN model and image resolution}

As noted in Sec. 1, convolution of the CLEAN model image with the CLEAN beam induces resolution loss in the final CLEAN image (and thus the 1D brightness profile). As an example, convolution of a circular beam whose full-width-at-half-maximum $(F W H M)$ is equal to the FWHM of a Gaussian feature in a brightness profile in a broadening of the feature by $\approx 40 \%$ and a reduction in its amplitude by $\approx 30 \%$. Convolution in real space corresponds to multiplication in Fourier space, which induces a loss in resolution in the visibility domain via an underestimate of the observed visibility amplitudes, an effect that worsens with baseline. The FWHM of a Gaussian in real space as a function of radius $r$ corresponds to a FWHM in Fourier space as a function of spatial frequency $q$ by
$\mathrm{FWHM}_{\mathrm{q}}=4 \ln (2) /\left(\pi \mathrm{FWHM}_{\mathrm{r}}\right)$, obtained by relating the standard deviations in real and Fourier space.

While CLEAN beam convolution is the primary source of resolution loss in the CLEAN procedure, additional contributions can arise from, e.g., non-Gaussianity of the PSF (dirty beam). To assess the inherent performance of the CLEAN algorithm - the resolution prior to CLEAN beam convolution - it is thus useful to examine the CLEAN model image (the .model output of tclean). A brightness profile extracted from this image directly measures the algorithm's achievable resolution and can itself be used to quantify a source's emission features. Some real astrophysical flux may be missed because the final residual image has not been added to the model image, and the brightness profile is often noisy due to the model image's sparse composition. But the Fourier transform of a profile extracted from the model image can quantify how well the modeling framework in the CLEAN procedure fits the observed visibility distribution as a function of baseline.

To this end, Fig. 1 compares the brightness profiles extracted from the convolved CLEAN image and the CLEAN model, as well as the Fourier transform of these profiles, for the DSHARP observations of AS 209. The profiles identify the same features in Fig. 1(a), but the CLEAN model profile shows higher amplitudes (though also more noise) and narrower widths for the two innermost disc features. This resolution advantage is not maintained across all disc features, as the CLEAN model profile does not recover the rings in the CLEAN image profile at $\approx 0.25^{\prime \prime}$ and $0.33^{\prime \prime}$. This is because not all of the real flux in the dirty image is incorporated into the CLEAN model. The CLEAN model profile also shows effectively identical widths and amplitudes as the CLEAN image profile for the two outer disc rings. Additionally and importantly, the CLEAN model can have negative components.

The Fourier domain equivalents of these brightness profiles in panel (b) show how the transform of the CLEAN image profile underestimates visibility amplitudes with increasing severity as baseline increases, as expected from beam convolution. The transform of the CLEAN model critically still underestimates the visibility amplitudes between $\approx 1.6-3.7 \mathrm{M} \lambda$, and overestimates amplitudes between $\approx 4.1-5.1 \mathrm{M} \lambda$. This demonstrates that additional factors beyond CLEAN beam convolution are nontrivially limiting recovery of the full information content in the long baseline data, and thus that the inherent performance of the CLEAN modeling framework is resolution-limited. We emphasize that all DSHARP datasets were CLEANed by experts in the field (Andrews et al. 2018; Huang et al. 2018a); these results trace practical resolution limits of CLEAN rather than the capability of a user.

For reference, if we compare the observed visibilities for a given survey dataset to the Fourier transform of a brightness profile extracted from the CLEAN image, then convolve the data with a beam that minimizes the difference with the Fourier transform of the brightness profile, the average CLEAN beam width across the survey is increased by a factor of 1.16 . This simplistically treats all resolution-limiting factors in the CLEAN images as convolution operators, but it gives a sense of the aggregate resolution limitations in the CLEAN images beyond the effect of CLEAN beam convolution. PSF sidelobe structure and the compromise between resolution and sensitivity in the choice of the Briggs robust parameter in tclean are two notable resolution-limiting contributors.

For comparison to the CLEAN image and CLEAN model profiles, the frank fit to AS 209 is also shown in Fig. 1. The frank profile in panel (a) more highly resolves features seen in the CLEAN image profile and suggests a small bump at $\approx 0.16^{\prime \prime}$ not present in either the CLEAN image profile or the CLEAN model profile. In panel 

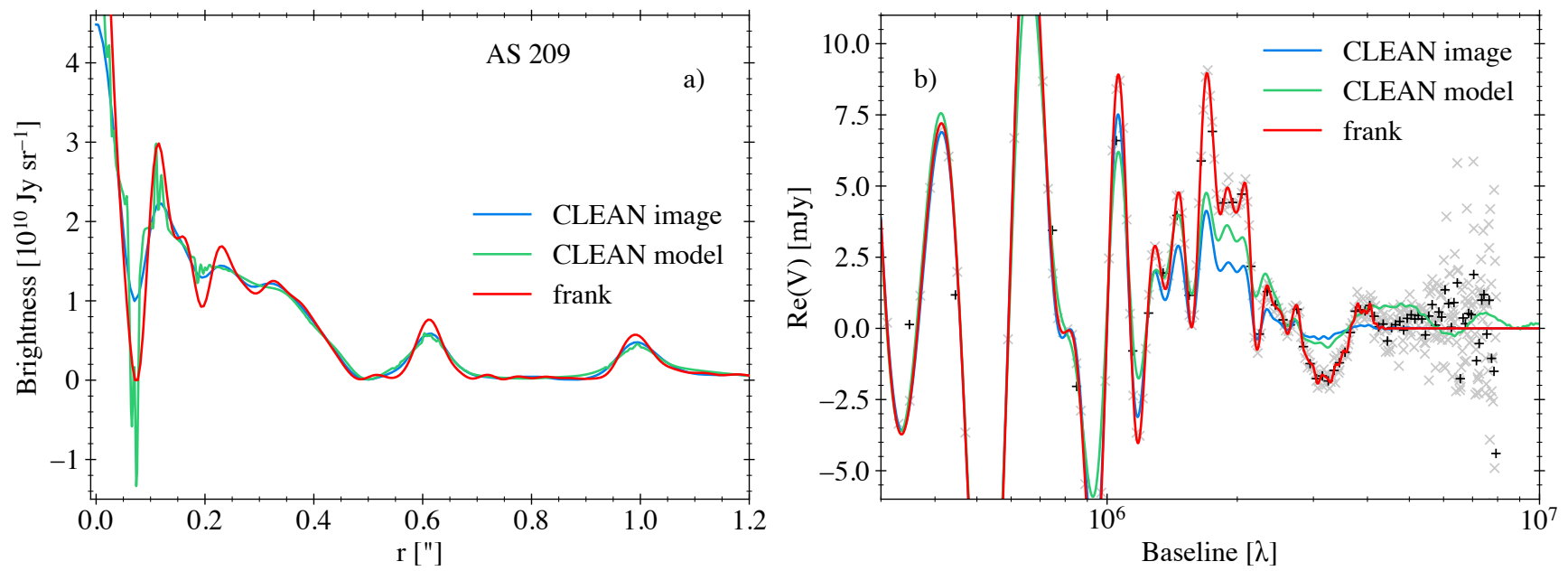

Figure 1. Effect of CLEAN beam convolution (and other factors) on substructure recovery in DSHARP

a) For the DSHARP observations of SR 4, radial brightness profiles extracted from the CLEAN image and CLEAN model, as well as the frank brightness profile. The frank profile and CLEAN model profile peak at 8 and $16 \times 10^{10} \mathrm{Jy} \mathrm{sr}^{-1}$ respectively.

b) The discrete Hankel transform (DHT) of the CLEAN profiles in (a), and the frank visibility fit. Data are shown in 20 and $100 \mathrm{k} \lambda$ bins.

(b), the frank visibility fit is correspondingly more accurate than the transforms of both the CLEAN profile and the CLEAN model beyond $\approx 1 \mathrm{M} \lambda$; factors problematic for CLEAN such as PSF sidelobe structure are not limiting the frank fit resolution. frank is thus outperforming the inherent resolution capability of the CLEAN algorithm. This relative performance holds across the DSHARP survey, as we will now quantify.

\subsection{Using the visibilities to quantify the accuracy of CLEAN model, CLEAN image, and frank brightness profiles}

It is useful to consider a metric that directly quantifies the accuracy of a 1D brightness profile extracted from a CLEAN image or CLEAN model by comparing the Fourier transform of the profile to the observed visibilities. Such a metric can incorporate all sources of error in the visibility domain representation of the profile, while being agnostic to the causes of these errors. This metric also allows us to compare the fit accuracy in CLEAN and frank. We will use as a metric a simple assessment of a profile's visibility space residuals.

We have found the most robust definition of a visibility space accuracy metric to be the shortest baseline $B_{80}$ beyond which a fit shows $\geq 20 \%$ error in visibility amplitude for a minimum consecutive $200 \mathrm{k} \lambda$ (using $20 \mathrm{k} \lambda$ binning). In practice these criteria robustly identify, across all 20 DSHARP sources, the first baseline at which the Fourier transform of a profile extracted from a CLEAN image or model, or the frank visibility fit, departs appreciably from the observed visibility amplitudes and only becomes more inaccurate with increasing baseline. Varying the $20 \%$ threshold has a weak effect on $B_{80}$, frank, while decreasing the threshold to $10 \%$ yields an average $B_{90}$, CLEAN image $=0.64 B_{80}$, CLEAN image, and $B_{90}$, CLEAN model $=0.87 B_{80}$, CLEAN model across the 20 DSHARP datasets. Increasing the threshold to $50 \%$ gives an average $B_{50}$, CLEAN image $=1.97 B_{80}$, CLEAN image and $B_{50}$, CLEAN model $=2.26 B_{80}$, CLEAN model . Varying the $200 \mathrm{k} \lambda$ threshold has a weak effect on $B_{80}$, frank, $B_{80}$, CLEAN image and

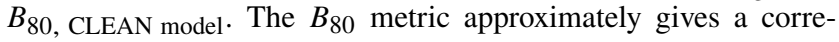
sponding spatial scale down to which a CLEAN or frank brightness profile accurately recovers substructure widths and amplitudes. A profile can of course partially recover information on smaller spatial scales, but features on these scales will be underresolved relative to the dataset's available resolution information.

Fig. 2(c) - (d) show the application of the $B_{80}$ accuracy metric to the Sz 129 DSHARP dataset. In panel (c) the Fourier transform of a brightness profile extracted from the CLEAN image has some small error prior to $B_{80}$, CLEAN image, while beyond this baseline the Fourier domain representation is, and remains, visibly inaccurate. The transform of a profile extracted from the CLEAN model has a $B_{80}$, CLEAN model that is highly similar to $B_{80}$, CLEAN image, with clear inaccuracy beyond this baseline. Applying the same metric to determine $B_{80}$, frank, the frank visibility fit in Fig. 2(c) accurately matches the observed visibility amplitudes out to $\approx 2.8 \mathrm{M} \lambda$, the baseline at which the binned data's SNR begins to oscillate about $\mathrm{SNR}=1$. Finally, the CLEAN (image and model) and frank residual visibilities in Fig. 2(d) demonstrate the higher accuracy of the frank fit even at moderate baselines. The CLEAN model residuals increase over a broad baseline range due to fundamental limitations in the CLEAN algorithm, while the CLEAN image residuals similarly increase over a broad range due additionally to CLEAN beam convolution. The frank residuals remain $\approx 0$ until the sharp rise at the baseline where the fit's SNR threshold is met and the fit drives toward zero.

The ordering of the baseline accuracy measurements for Sz 129 is indicative of results across the survey: $B_{80}$, CLEAN image $\lesssim$

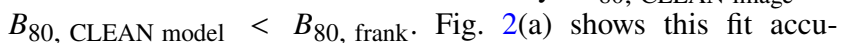
racy analysis for all DSHARP sources, ordered by increasing $B_{\text {data, expected }}$, the baseline equivalent of the expected angular resolution,

$\theta_{\text {data, }}$ expected $=0.574 \lambda / L_{80}$.

Here $\lambda$ is the observing wavelength and $L_{80}$ is the eightieth percentile of the baseline distribution (Remijan et al. 2019). For reference, the observed visibility distributions for the DSHARP datasets typically extend to $\approx 8-10 \mathrm{M} \lambda$, with a mean $B_{\text {data, expected }}=4.72$ $\mathrm{M} \lambda$. Fig. 2(b) shows that across the 20 DSHARP datasets, frank is accurately fitting the visibilities to a mean factor of 4.3 longer baseline than brightness profiles extracted from the CLEAN images, and 


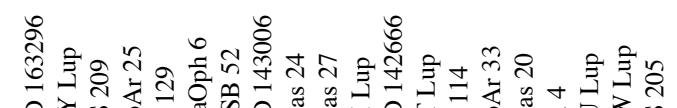

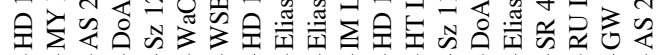

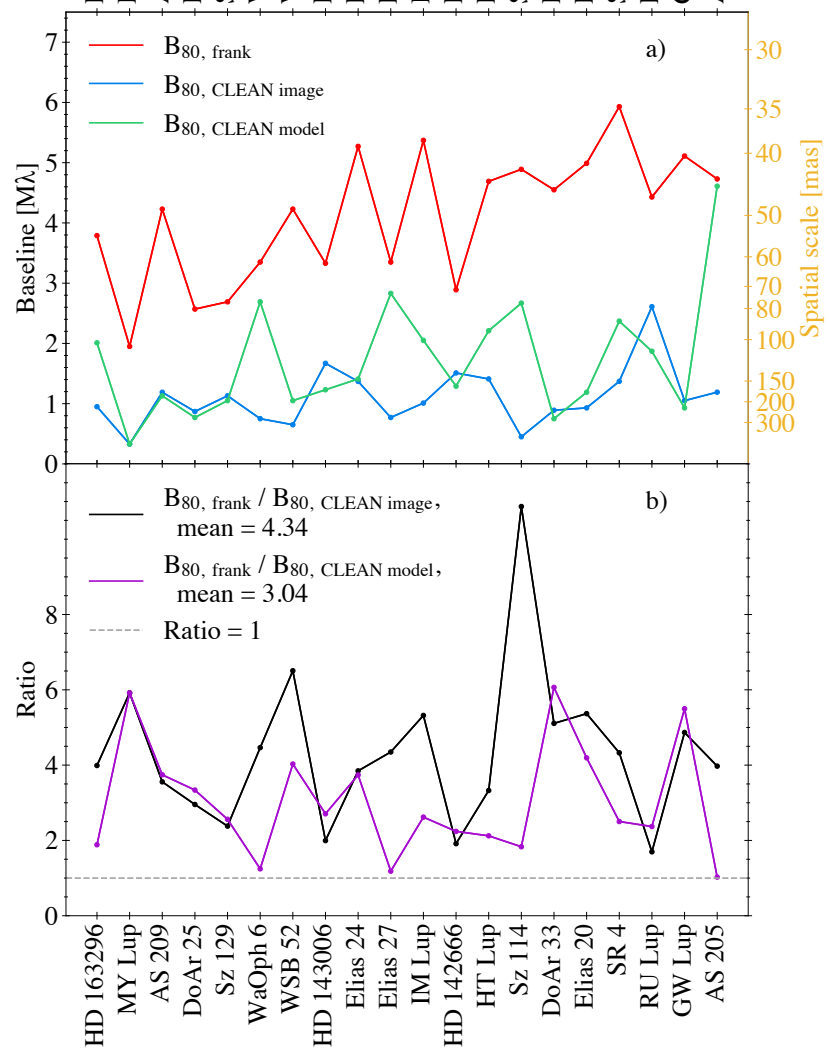

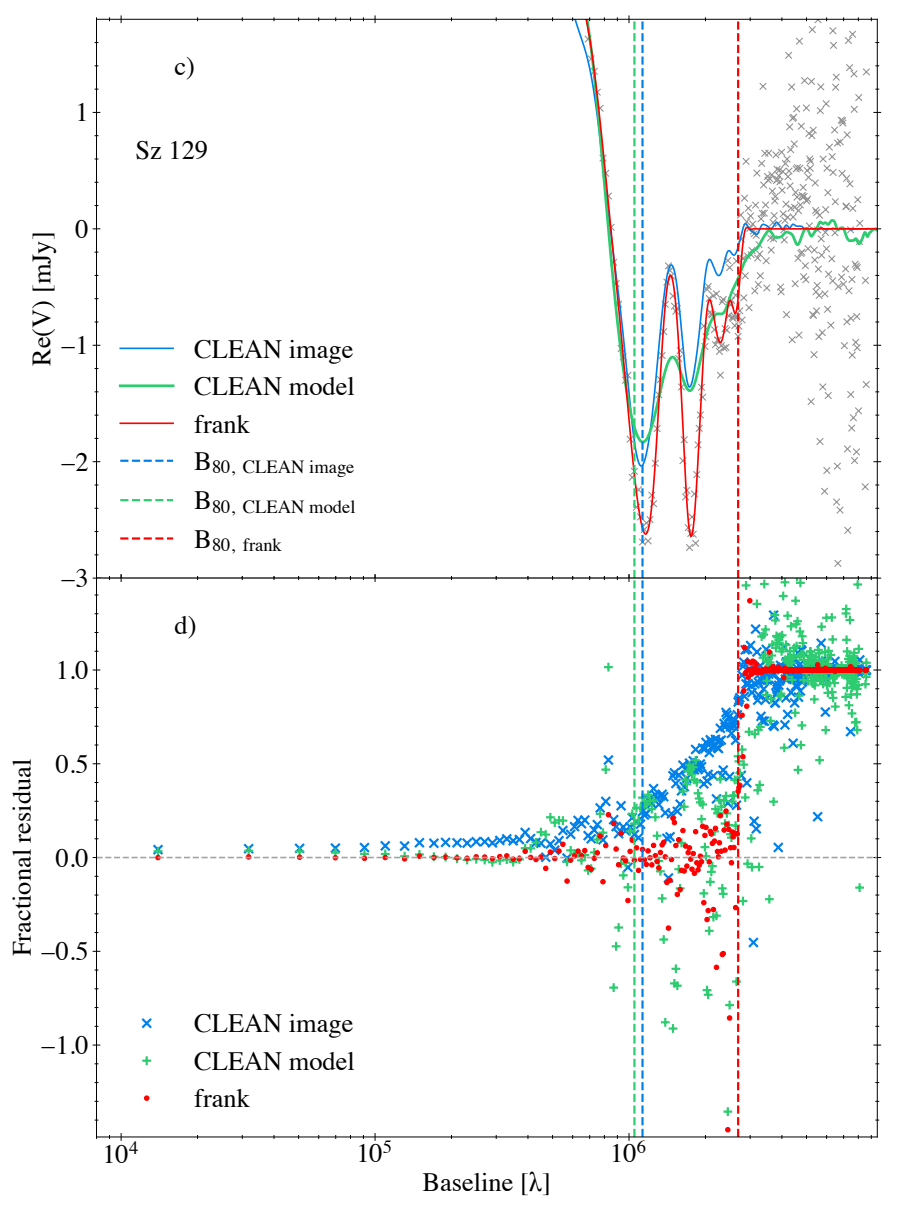

Figure 2. CLEAN and frank fit accuracies in DSHARP

a) Baseline accuracy metric $\boldsymbol{B}_{80}$ for the convolved CLEAN image, CLEAN model, and frank visibility fits across the 20 DSHARP sources. The accuracy metric is the shortest baseline beyond which a fit shows $\geq 20 \%$ error in visibility amplitude for a consecutive $\geq 200 \mathrm{k} \lambda$ (Sec. 3.2 ). Sources are sorted by the expected baseline resolution of each dataset (see Equation 1).

b) Ratio of the frank to CLEAN baseline accuracy metric for both the convolved CLEAN image and CLEAN model visibility fits.

c) An example of the baseline accuracy calculation. The visibility distribution for $\mathrm{Sz} 129$ (20 k $\lambda$ bins), the frank visibility fit, and the Fourier transform of the brightness profiles extracted from the convolved CLEAN image and CLEAN model.

d) Fractional residuals [(data - model / data); $20 \mathrm{k} \lambda$ bins] for the convolved CLEAN image visibility fit, CLEAN model visibility fit and frank visibility fit.

Table 1. Expected and achieved fit accuracy metrics shown in Fig. 2, as well as the baseline equivalent of the data's expected resolution given in Equation 1

. Standard deviations assume a Gaussian distribution. Conversions to au account for the unique distance to each source. $\lambda$ is the observing wavelength; $L_{80}$ is the eightieth percentile of the baseline distribution. The last two rows give a mean and standard deviation taken across the 20 datasets (i.e., not simply the ratio of preceding rows).

\begin{tabular}{lc}
\hline Baseline quantity, $B$ & Mean and standard deviation \\
\hline $\boldsymbol{B}_{\text {data, expected }}=0.574 \lambda / L_{80}$ & $4.75 \pm 1.39 \mathrm{M} \lambda$ \\
$\boldsymbol{B}_{80, \text { CLEAN image }}$ & $1.10 \pm 0.48 \mathrm{M} \lambda$ \\
$\boldsymbol{B}_{80, \text { CLEAN model }}$ & $1.72 \pm 0.97 \mathrm{M} \lambda$ \\
$\boldsymbol{B}_{80, \text { frank }}$ & $4.12 \pm 1.05 \mathrm{M} \lambda$ \\
$\boldsymbol{B}_{80, \text { frank }} / \boldsymbol{B}_{80, \text { CLEAN image }}$ & $4.34 \pm 1.99$ \\
$\boldsymbol{B}_{80, \text { frank }} / \boldsymbol{B}_{80, \text { CLEAN model }}$ & $3.04 \pm 1.47$ \\
\hline
\end{tabular}

a factor 3.0 longer baseline than profiles extracted from the CLEAN models. This reaffirms that frank is outperforming the achieved resolution in both the CLEAN images and CLEAN models. The resolution ratios and individual fit metrics are summarized in Table 1.
For reference, increasing the accuracy metric's error threshold from $20 \%$ to $50 \%$ decreases the mean $B_{80}$, frank $/ B_{80}$, CLEAN image from 4.3 to 3.0 , and the mean $B_{80}$, frank $/ B_{80}$, CLEAN model from 3.0 to 1.9 .

\subsection{A general note on comparing frank to CLEAN}

The CLEAN algorithm is a model to deconvolve the $2 \mathrm{D}$ sky brightness from the instrument PSF, which requires a functional form for the fundamental brightness unit (e.g., point sources or Gaussians). By comparison, frank is a visibility fitter, with the express goal of accurately recovering the $1 \mathrm{D}$ projection of the data. This is done nonparametrically, but requires assumptions that the emission is axisymmetric and that the source geometry can be perfectly determined. These two tools can be used for different goals; in the case of accurately describing a source's azimuthally averaged brightness, frank offers a clear resolution advantage over a profile extracted from a CLEAN image. The tradeoff is the potential imprint of reasonably high contrast azimuthal asymmetries on the morphology of a frank brightness profile; this must be diagnosed by Fourier 
Table 2. For each DSHARP source, values for the five hyperparameters used to produce the frank fit: SNR criterion $\alpha$, strength of smoothing $w_{\text {smooth }}$ applied to the reconstructed power spectrum, outer radius of the fit $R_{\text {out }}$, number of radial and spatial frequency points $N$ used in the fit, and floor value $p_{0}$ for the reconstructed power spectral mode amplitudes. Sensible choices for $R_{\text {out }}, N$ and $p_{0}$ have a trivial effect on the fits: $R_{\text {out }}$ is chosen to be larger than the disc's outer edge, $N$ is increased proportionally to $R_{\text {out }}$, and $p_{0}$ is the same for all fits. Sec. 2 gives a fuller explanation of, and motivation of the values for, $\alpha$ and $w_{\text {smooth. }}$. Some fits, as indicated, are forced to be nonnegative or are fit with a combined frank and unresolved component model (in which case the visibility offset applied for the unresolved component is given); see Sec. 2.1-2.2. In the rightmost column, sources whose imaged frank residuals show the brightness asymmetry discussed in Sec. 5.7 are noted. All frank fits are available at https://zenodo.org/record/5587841.

\begin{tabular}{|c|c|c|c|c|c|c|c|}
\hline Disc & $\alpha$ & $\log _{10} w_{\text {smooth }}$ & $\left.R_{\text {out }}{ }^{\prime \prime}\right]$ & $N$ & $p_{0}\left[\mathrm{Jy}^{2}\right]$ & Fit conditions & Brightness asymmetry \\
\hline AS 205 & 1.05 & -1 & 2.2 & 457 & $10^{-15}$ & & \\
\hline AS 209 & 1.05 & -4 & 1.9 & 395 & $"$ & Nonnegative fit & $\checkmark$ \\
\hline DoAr 25 & 1.05 & -1 & 3.1 & 500 & " & Unresolved component fit; offset $0.24 \mathrm{mJy}$ & \\
\hline DoAr 33 & 1.01 & -4 & 0.5 & 150 & $"$ & Unresolved component fit; offset $0.51 \mathrm{mJy}$ & $\checkmark$ \\
\hline Elias 20 & 1.01 & -4 & 1.1 & 222 & " & Unresolved component fit; offset $0.66 \mathrm{mJy}$ & \\
\hline Elias 24 & 1.01 & -4 & 1.9 & 395 & " & Unresolved component fit; offset $0.95 \mathrm{mJy}$ & $\checkmark$ \\
\hline Elias 27 & 1.25 & -1 & 2.9 & 500 & " & Unresolved component fit; offset $0.40 \mathrm{mJy}$ & $\checkmark$ \\
\hline GW Lup & 1.05 & -1 & 1.4 & 296 & $"$ & Unresolved component fit; offset $0.73 \mathrm{mJy}$ & $\checkmark$ \\
\hline HD 142666 & 1.50 & -4 & 0.7 & 150 & $"$ & & $\checkmark$ \\
\hline HD 143006 & 1.01 & -3 & 0.8 & 173 & $"$ & & \\
\hline HD 163296 & 1.01 & -4 & 2.9 & 500 & $"$ & & $\checkmark$ \\
\hline HT Lup & 1.05 & -3 & 0.6 & 150 & $"$ & & \\
\hline IM Lup & 1.10 & -1 & 2.4 & 494 & $"$ & Unresolved component fit; offset $0.46 \mathrm{mJy}$ & $\checkmark$ \\
\hline MY Lup & 1.01 & -4 & 1.2 & 247 & " & Unresolved component fit; offset $0.26 \mathrm{mJy}$ & \\
\hline RU Lup & 1.05 & -4 & 0.7 & 150 & $"$ & & $\checkmark$ \\
\hline SR 4 & 1.05 & -4 & 0.5 & 150 & $"$ & & \\
\hline Sz 114 & 1.05 & -2 & 0.7 & 150 & $"$ & Unresolved component fit; offset $0.51 \mathrm{mJy}$ & \\
\hline Sz 129 & 1.50 & -4 & 1.0 & 198 & $"$ & Nonnegative fit & \\
\hline WaOph 6 & 1.01 & -4 & 1.9 & 395 & $"$ & Unresolved component fit; offset $0.83 \mathrm{mJy}$ & $\checkmark$ \\
\hline WSB 52 & 1.01 & -4 & 0.5 & 150 & " & Unresolved component fit; offset $0.33 \mathrm{mJy}$ & \\
\hline
\end{tabular}

Table 3. Major new and appreciably more highly resolved features identified in frank brightness profiles. Feature widths and gap depths are defined following the method in Huang et al. (2018a); see Sec. 5. The datasets' finite resolution entail that the values for ring widths are upper bounds, and for gap widths and depths are lower bounds.

\begin{tabular}{|c|c|c|c|c|c|}
\hline Disc & $\begin{array}{l}\text { New (or better resolved) } \\
\text { feature }\end{array}$ & $\begin{array}{l}\text { Location } \\
\text { [au] }\end{array}$ & $\begin{array}{c}\text { Identifier in } \\
\text { Huang et al. (2018a) }\end{array}$ & $\begin{array}{c}\text { Width [au] (width in } \\
\text { Huang et al. 2018a [au]) }\end{array}$ & $\begin{array}{l}\text { Gap depth (depth in } \\
\text { Huang et al. 2018a) }\end{array}$ \\
\hline \multirow[t]{2}{*}{ AS 209} & Deeper gap, & 9 & D9 & $5.2(4.7 \pm 0.2)$ & $0.00(0.45 \pm 0.02)$ \\
\hline & brighter ring & 14 & B14 & $4.9(8.9 \pm 0.2)$ & N/A \\
\hline Elias 24 & New gap & 14 & - & $2.2(-)$ & $0.89(-)$ \\
\hline \multirow[t]{2}{*}{ GW Lup } & Deeper and sharper gap, & 75 & D74 & $11.7(12.1 \pm 0.4)$ & $0.01(0.31 \pm 0.03)$ \\
\hline & brighter and narrower ring & 85 & B85 & $7.5(11.3 \pm 0.4)$ & N/A \\
\hline \multirow{2}{*}{ HD 142666} & New gap, & 3 & - & $3.6(-)$ & $0.42(-)$ \\
\hline & brighter ring & 7 & B6 & $4.0(5.3 \pm 1.4)$ & N/A \\
\hline \multirow[t]{5}{*}{ HD 143006} & Cleared inner cavity, & $\leq 7$ & - & N/A & N/A \\
\hline & brighter rings, & $7,41,64$ & B6, B41, B65 & $5.3,5.4,9.5(5.0 \pm 1.4,12.2 \pm 1.0,11.5 \mathrm{pm} 1.4)$ & N/A \\
\hline & wider and sharper gaps ${ }^{\dagger}$ & 25,52 & D22, D51 & $28.4,16.1$ & $0.07,0.43$ \\
\hline & & & & $(21.7 \pm 1.0,12.8 \pm 1.4)$ & $(0.04 \pm 0.02,0.53 \pm 0.02)$ \\
\hline & brighter and narrower ring & 41 & B41 & $5.4(12.2 \pm 1.0)$ & N/A \\
\hline \multirow[t]{2}{*}{ HD 163296} & Deeper gap, & 10 & D10 & $3.0(3.2 \pm 1.4)$ & $0.47(0.93 \pm 0.03)$ \\
\hline & brighter ring & 13 & B14 & $3.8(3.6 \pm 1.4)$ & N/A \\
\hline RU Lup & Deeper gaps & $14,21,29$ & D14, D21, D29 & $\begin{array}{c}3.1,3.4,4.8 \\
(-,<7,4.5 \pm 0.3)\end{array}$ & $\begin{array}{c}0.90,0.75,0.57 \\
(-,-, 0.78 \pm 0.01)\end{array}$ \\
\hline \multirow[t]{2}{*}{ SR 4} & New gap, & 4 & - & $1.4(-)$ & $0.64(-)$ \\
\hline & wider and deeper gap ${ }^{\dagger}$ & 11 & D11 & $8.6(6.3 \pm 1.4)$ & $0.02(0.23 \pm 0.02)$ \\
\hline \multirow[t]{2}{*}{ Sz 129} & Cleared inner cavity, & $\leq 11$ & - & N/A & N/A \\
\hline & brighter ring & 11 & B10 & $12.3(17.6 \pm 1.1)$ & N/A \\
\hline
\end{tabular}

$\dagger$ Because these gaps are structured in the frank profiles, the gap center is determined as the average of the adjacent ring centers. The gap depth is

determined using the average brightness across the gap width.

transforming (imaging) the residual frank visibilities and/or examining the imaginary component of the observed data. In summary, for the purpose of obtaining a $1 \mathrm{D}$ brightness profile of a source (under the assumptions of axisymmetry and known source geometry), frank will yield a more accurate (higher resolution) result, without a loss in sensitivity, compared to extracting an azimuthally averaged profile from the CLEAN image.

\section{RESULTS}

Fig. 3 shows the frank brightness profile for each DSHARP disc, as well as the CLEAN image profile from Huang et al. (2018a) and the CLEAN model profile obtained using the published tclean scripts. The frank fits exhibit more highly resolved, and in some cases new, substructure relative to the CLEAN images. Consistent with expecta- 

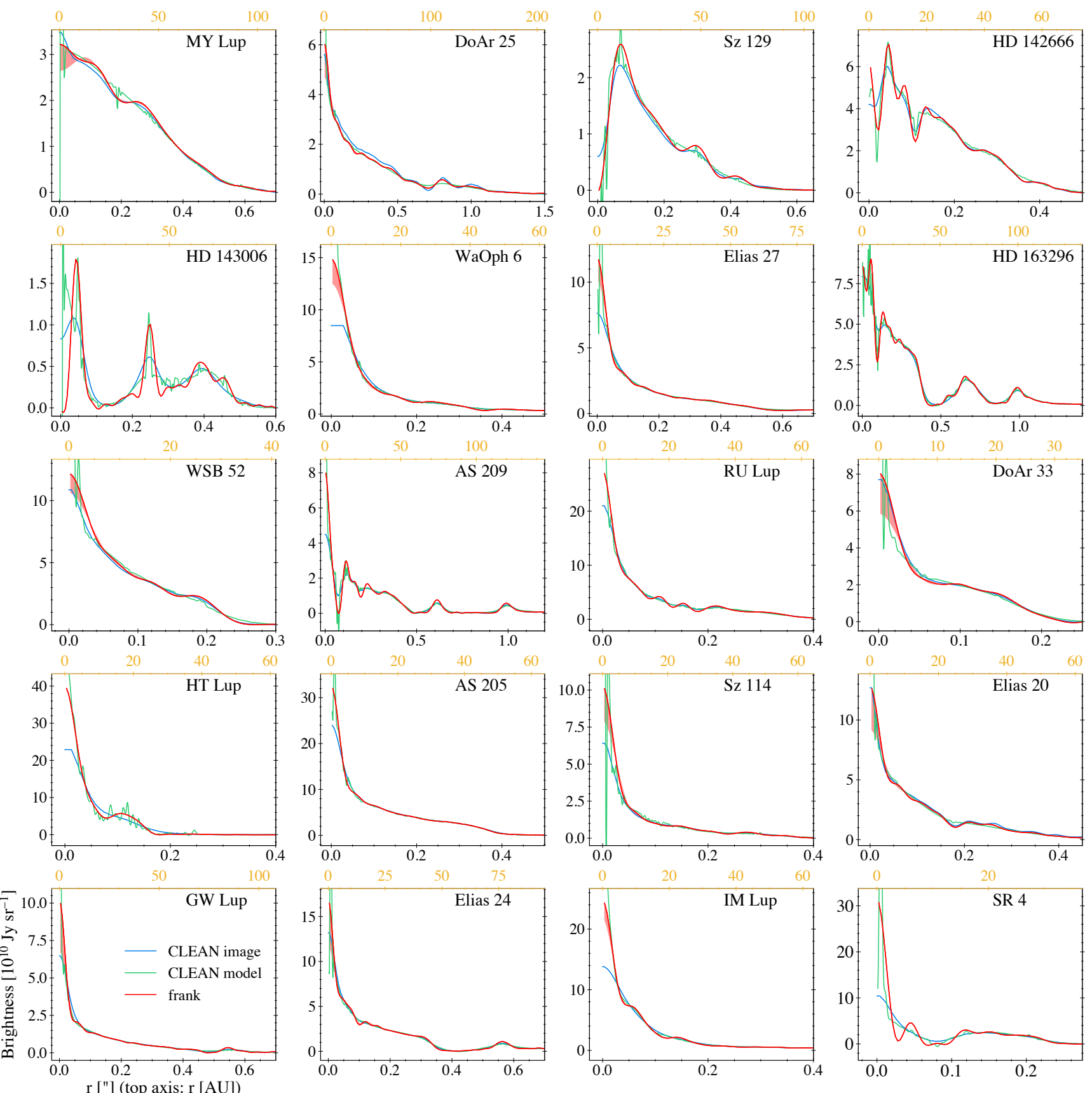

Figure 3. Recovered brightness profiles

For each source in the DSHARP survey, the convolved CLEAN image, CLEAN model and frank brightness profiles. Some profiles zoom on the inner region of the disc. Discs are arranged from left to right and then top to bottom in ascending order of frank fit resolution. Informal uncertainties are shown on discs fit with the point source-corrected model (Sec. 2.1).

tions from CLEAN beam convolution, the CLEAN image profiles also tend to underestimate the source's peak brightness (frank must as well, albeit to a lesser extent). The frank profiles further identify fine substructure more clearly than the noisy CLEAN model profiles. As a general note, feature morphologies primarily in the inner disc of the frank profiles can be expected to evolve with higher resolution observations, which could for example find gaps to be deeper and broader, resolve rings into multiple components, or reduce the amplitude of features by placing stronger constraints on structure at the smallest scales recovered in these data. Table 2 gives the values of the hyperparameters used in each frank fit.

Fig. 4 shows a zoom on the long baselines of the frank visibility fits and the Fourier transform of the CLEAN image and model brightness profiles across the survey. The higher resolving power evident in the frank brightness profiles for all 20 sources corresponds to the frank visibility fits matching the data at high accuracy to longer baseline than the CLEAN image profiles and (to a lesser extent) the CLEAN model profiles. Table 2 notes which frank fits use the 

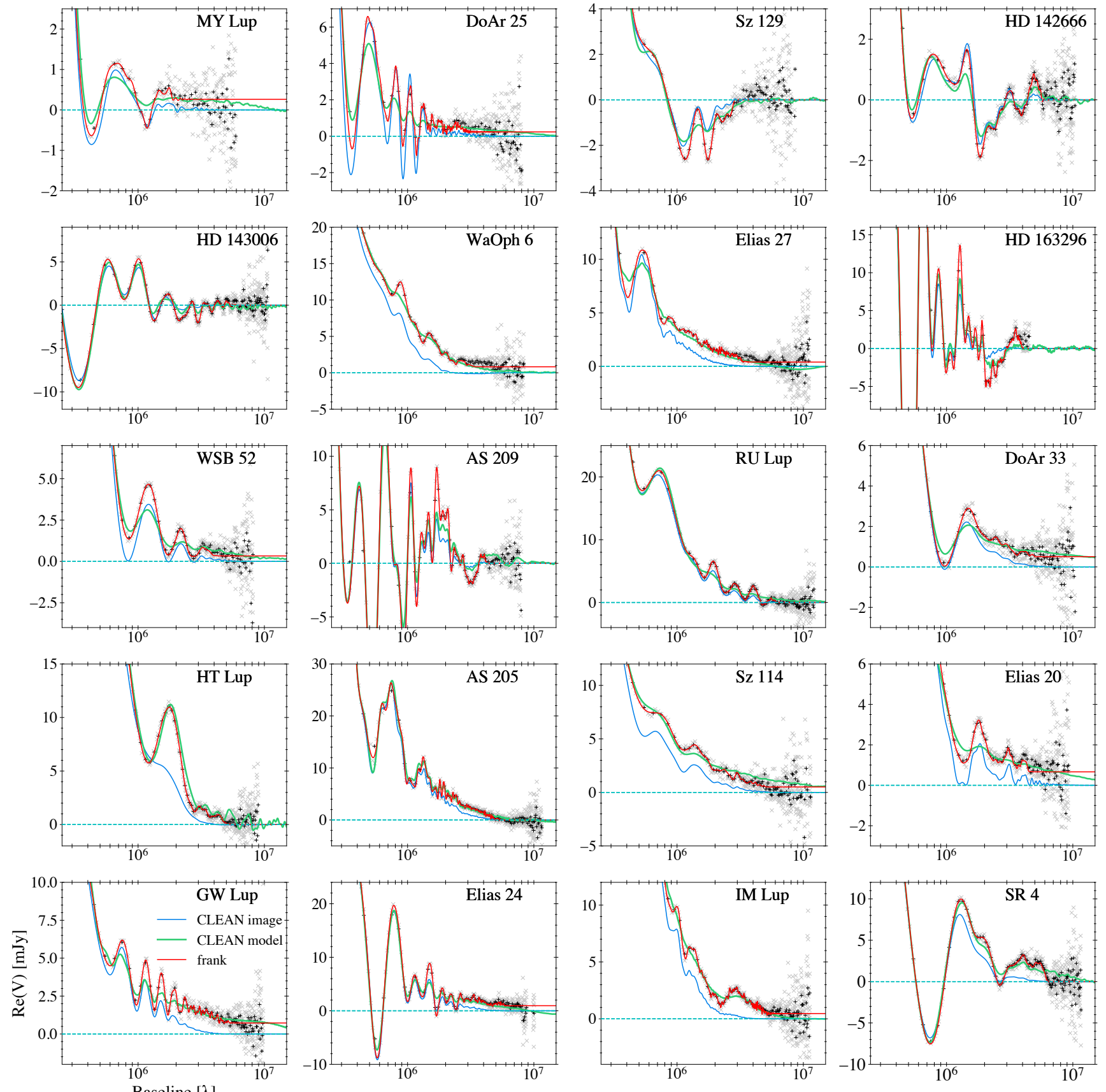

Figure 4. Visibility fits at long baseline

For each source in the DSHARP survey, a zoom on the data's long baselines ( $>0.25 \mathrm{M} \lambda$, corresponding to spatial scales $<0.83^{\prime \prime}$ mas) to show the accuracy of the CLEAN image, CLEAN model and frank fits in matching detailed visibility structure. Data are shown in 20 and $100 \mathrm{k} \lambda$ bins and become heavily noise-dominated at the longest baselines across all datasets, typically at $\gtrsim 5 \mathrm{M} \lambda$. frank does not fit these regions, as doing so would imprint noisy oscillations on the recovered brightness profile. Discs are arranged from left to right and then top to bottom in ascending order of frank fit resolution.

point source-corrected model (Sec. 2.1) and gives the point source visibility amplitude applied. For some sources - DoAr 25, Elias 27, HD 163296, AS 205, GW Lup, Elias 24, and IM Lup - frank is clearly fitting some noise on top of the signal at long baseline. This manifests as short spatial period, low amplitude $(<1 \%$ of the peak brightness) noise in the corresponding brightness profile. We accept this as a tradeoff for fitting out to baselines at which the binned data SNR approaches unity. The effect is seen most clearly in the logarithmic brightness plots for GW Lup, Elias 24 and HD 163296 in Fig. 10 (which will be discussed in Sec. 5.6).

\section{ANALYSIS}

Table 3 summarizes the major new and appreciably better resolved annular features in the frank fits across the survey, as well as 
quantifies the gap/ring widths and gap depths. For the purpose of comparison, this quantification follows the approach in Huang et al. 2018a (see their §3.2). The metric measures a gap depth as the ratio of the brightness at center of the gap $I_{\mathrm{d}}$ to the brightness at the center of the ring $I_{\mathrm{b}}$ exterior to the gap, and determines a feature width by defining the edges of an adjacent gap and ring using the average $I_{\text {mean }}=0.5\left(I_{\mathrm{d}}+I_{\mathrm{b}}\right)$. This does not yield a perfect comparison for feature widths and depths between CLEAN and frank profiles, because the frank profiles exhibit additional low amplitude substructure (e.g., in some gaps and on the wings of some rings). But as a coarse comparison, among the features in Table 3 , 7 of the 12 gaps and each of the 8 rings were quantified in Huang et al. (2018a). For this subset, the frank profiles find the gaps to be a mean $14 \%$ wider and $44 \%$ deeper, and the rings to be a mean $26 \%$ narrower. This illustrates the utility of the super-resolution fits for substructure characterization.

Grouping the frank brightness profiles in Fig. 3 by morphology, we can identify new substructure trends. We will exclude the multiple systems HT Lup and AS 205 from the following analysis because, as discussed in Sec. 2.2, while the 1D frank profiles are not visibly biased by the presence of multiple sources in the field of view, application of the model to such a case is still formally incorrect. We do note here that the frank fit for HT Lup identifies the primary disc's spiral structure as the bump in the profile at 15 au in Fig. 3.

Collectively, these trends as detailed below demonstrate two broad findings. First, the DSHARP sources - already rife with gaps and rings as identified in Huang et al. (2018a) - are even more structured, especially interior to 30 au. Second, the gaps and rings detected in the CLEAN images, which in many cases have widths $2-3 \times$ that of the CLEAN beam, become deeper and wider (gaps) or narrower and brighter (rings) when we fit the data with frank.

\subsection{The compact DSHARP discs all show substructure}

The super-resolution frank fits find new substructure in each of the DSHARP survey's three compact $\left(R_{\max }<50 \mathrm{au}\right)$, single-disc systems - WSB 52, DoAr 33 and SR 4. As a prominent example - shown in Fig. 5 - the frank profile for SR 4 resolves the broad depression in the CLEAN profile into two distinct, deep gaps within 20 au (those listed in Table 3). The innermost of these is centered at $4 \mathrm{au}$; the outer, centered at $11 \mathrm{au}$, is predicted by frank to be at least as deep as the fit's noise floor $\left(\approx 10^{9} \mathrm{Jy} \mathrm{sr}^{-1}\right.$, or $4 \%$ of the fitted peak brightness). Additionally, the frank profile for WSB 52 finds a new, shallow gap/ring pair at 13/17 au (in addition to the previously identified gap/ring pair at 21/25 au), and the frank fit for DoAr 33 resolves the single gap/ring pair at 9/17 au in the CLEAN profile into two gap/ring pairs.

Typical of current observations of compact discs, the shallow features in the frank profiles for these compact sources could be either intrinsically wide and shallow or narrow and underresolved. Sensitive observations at higher angular resolution are needed to distinguish between the two scenarios. We use a point source-corrected fit for WSB 52 and DoAr 33 (Sec. 2.1), with the profile's sensitivity to the point source visibility amplitude shown as the informal uncertainty band in Fig,. 5. The substructure in both sources is robust to this informal uncertainty.

The commonality of substructure frank finds across these three compact DSHARP sources suggests that in general compact discs, just as more extended discs, may routinely exhibit annular substructure. SR 4 is particularly notable in this context, with its effectively empty gap at 11 au analogous to the empty gap frank finds at 10 au in the much larger disc of AS 209 (outer radius $\approx 150$ $\mathrm{au}$ ). If compact discs are frequently structured, it may follow that the same physical processes (including companions) responsible for structure in larger discs are also efficacious in smaller discs. The improved identification of substructure in the compact DSHARP discs is also of particular interest, as compact sources represent a significant yet understudied component of the protoplanetary disc population.

\subsection{Extended discs show brighter rings, deeper gaps, and hints of inner disc substructure}

frank fits for several extended DSHARP sources better resolve the gaps and rings that appear shallow in the CLEAN profiles, as shown in Fig. 6. This is especially apparent in the outer gap and ring pair in GW Lup, where in the frank profile the brightness contrast between the gap and ring is 0.01 , compared to 0.31 in the CLEAN profile (see Table 3); and in RU Lup, where the three consecutive gaps interior to $30 \mathrm{au}$ are deeper in the frank profile (the contrast of the gap at 29 au for example is 0.57 in the frank fit, compared to 0.78 in the CLEAN profile). The frank fit to Elias 24 robustly finds a new gap at $13 \mathrm{au}$, and the model better resolves the faint ring at $45 \mathrm{au}$ in Sz 114.

For RU Lup, Sz 114, Elias 20, GW Lup, and Elias 24, the model suggests a steep inner disc in the inner $5-7$ au, followed by a shallower slope at slightly larger radii. This may be an indication of underresolved substructure between $\approx 7-12$ au. We use the point source-corrected fit (Sec. 2.1) for 5 of the 6 sources in Fig. 6 and show the profile's sensitivity to the point source visibility amplitude as the informal uncertainty band. This suggests we should be cautious about the fit's exact structure in the innermost disc, while the change in slope is robust to this uncertainty.

In addition to these sources, the frank brightness profile for a majority of the 20 DSHARP discs exhibits either gap and ring substructure interior to $30 \mathrm{au}$, or clear change in slope interior to $\approx$ $12 \mathrm{au}$. This suggests substructure is common not only at $\geq 30$ au, but also at the smaller separations that harbor the bulk of the observed exoplanet population. The Gaussian kernel density estimate for gap and ring locations in Huang et al. (2018a) peaks at 30 au, while by comparison the frank fits suggest that the occurrence rate continues to rise toward $r=0$. The (effectively) empty gaps at $\approx 10$ au in the frank fits for AS 209 (gap contrast of 0.00 in the frank profile, compared to 0.45 in the CLEAN profile) and SR 4 (contrast of 0.02 in the frank profile, compared to 0.23 in the CLEAN profile) suggest that the lack of such deep features identified thus far in high resolution disc observations is an artifact of resolving power, rather than an intrinsic absence of cleared gaps in inner discs.

\subsection{Two of the oldest DSHARP discs appear to have inner cavities}

frank finds that 2 of the 20 DSHARP discs, HD 143006 and Sz 129, have a fully cleared inner cavity. The CLEAN profiles for these sources show a decreasing brightness toward $r=0$, but not a full cavity in Fig. 7, and the frank fits also find the discs to have an appreciably brighter inner rim (noted in Table 3). Huang et al. (2018a) inferred the presence of a cleared cavity in these sources from the CLEAN images, now confirmed by the frank fits. The spectral energy distribution (SED) for HD 143006 (and potentially for Sz 129) shows a dearth in the near-IR $(\approx 10-20 \mu \mathrm{m})$ and excess in the far-IR $(\approx 20-100 \mu \mathrm{m})$ as shown in Fig. 7 (SEDs adapted from 

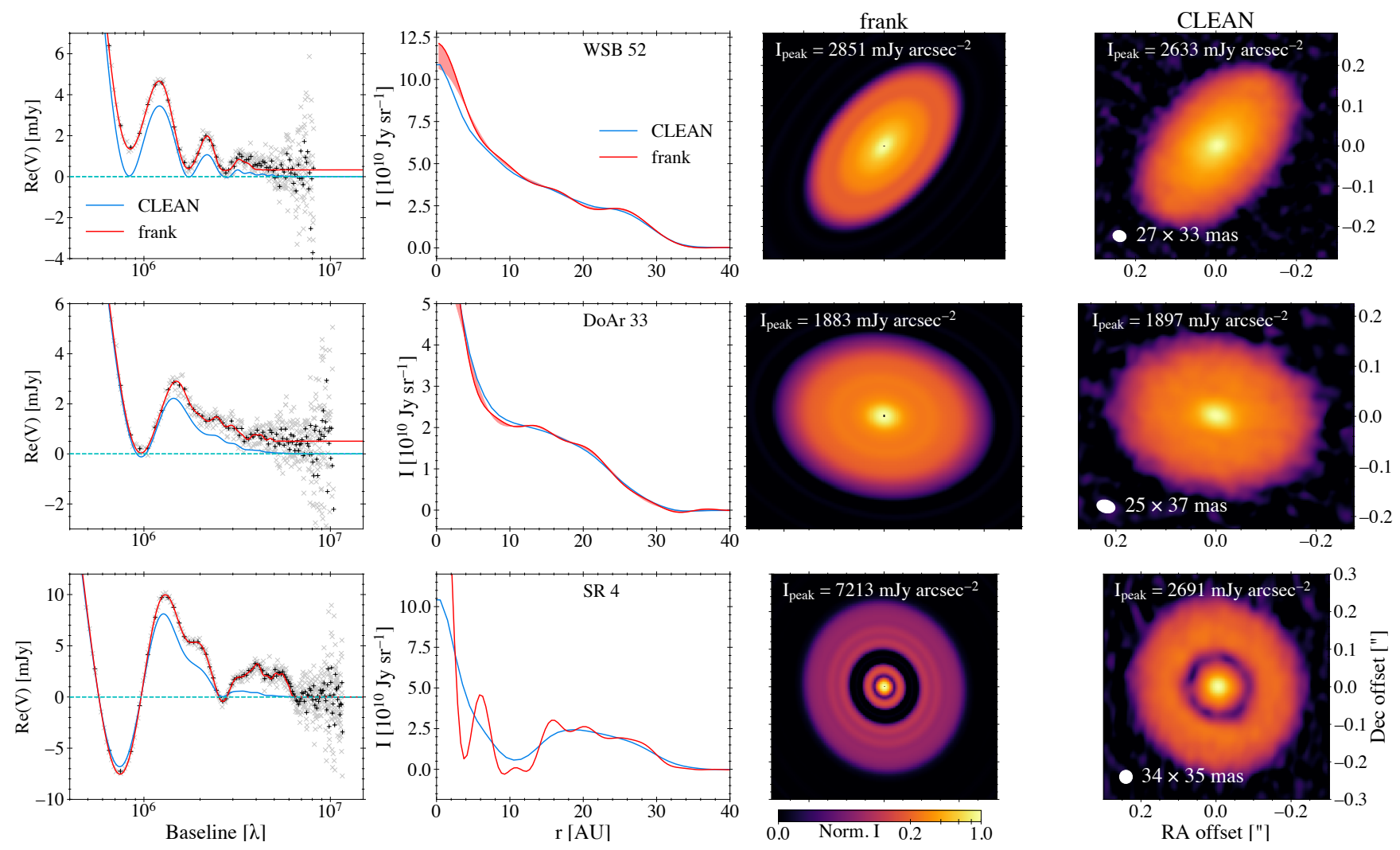

Figure 5. Substructure in compact discs

For each of the compact $\left(R_{\max }<50 \mathrm{au}\right)$ single-disc systems in DSHARP, a zoom on the data's long baselines ( $>0.40 \mathrm{M} \lambda$, corresponding to spatial scales $<0.52^{\prime \prime}$ mas; data shown in 20 and $100 \mathrm{k} \lambda$ bins), the frank and CLEAN visibility domain fits, the frank and CLEAN brightness profiles (in some cases zoomed into lower brightness), an image of the frank profile swept over $2 \pi$ and reprojected, and the CLEAN image. The frank and CLEAN images of each disc use the same $\operatorname{arcsinh}$ stretch $\left(I_{\text {stretch }}=\operatorname{arcsinh}(I / a) / \operatorname{arcsinh}(1 / a), a=0.02\right)$, but different brightness normalization. The generic color bar gives the normalized color scale, and the peak brightness is listed on each image. Discs are arranged from top to bottom by increasing frank fit resolution. Informal uncertainties are shown on discs fit with the point source-corrected model (Sec. 2.1).

Andrews et al. 2018). These may be indications of transition discs; it is also possible that either of these sources has a sharp rise in brightness in the innermost disc that is not resolved by frank.

Intriguingly, HD 143006 and Sz 129 may be two of the oldest discs in the DSHARP sample. Among the survey's single-disc systems, 5 of 18 orbit a star whose inferred age is $>2 \mathrm{Myr}$ as reported in Andrews et al. 2018 (see specific references in their Table 1): HD $143006(4.0 \pm 2.0 \mathrm{Myr}), \mathrm{Sz} 129(4.0 \pm 2.5 \mathrm{Myr})$, MY Lup $\left(10.0_{-2.0}^{+4.0} \mathrm{Myr}\right), \mathrm{HD} 142666(12.6 \pm 0.3 \mathrm{Myr})$, and HD 163296 $(12.6 \pm 4.0 \mathrm{Myr})$. These estimates are in general subject to systematic challenges such as interpreting robust ages at high effective temperature, and Andrews et al. (2018) additionally note that the age for MY Lup may be overestimated due to the inclined and flared disc extincting the stellar spectrum. Of the remaining four potentially old sources, HD 143006 and Sz 129 show inner cavities in the frank fits, while HD 142666 and HD 163296 both show gaps interior to $\approx 5$ au. No other frank brightness profile in DSHARP shows a turnover in brightness interior to $5 \mathrm{au}$, which may tentatively suggest that these four objects are experiencing the later stages of disc dispersal, losing (or having already lost) their inner disc at their potentially advanced ages. The expectation is in line with the finding by Espaillat et al. (2014) that the fraction of transition discs in star forming regions and young clusters increases from $\approx 1 \%$ to $\approx 10 \%$ for ages $\gtrsim 2 \mathrm{Myr}$ (these percentages do carry large uncertainties).
More speculatively, HD 142666, HD 143006 and HD 163296 are 3 of the 4 most structured discs in the survey, perhaps indicating that even if annular substructures do form early, discs may become more structured over time (e.g., as additional planets form). AS 209 complicates this interpretation though, being the other highly structured disc in the survey and having an inferred age of only $1.0_{-1.0}^{+2.5}$ Myr.

\subsubsection{Improved constraints on dust trapping}

The narrower rings in the frank fits relative to CLEAN can offer improved constraints on dust trapping. Dullemond et al. (2018) examine the outer disc rings in the CLEAN profiles for five of the DSHARP sources - AS 209, Elias 24, GW Lup, HD 143006, and HD 163296 - and infer deconvolved widths $w_{\text {dust }}$ to compare to the local pressure scale height $h_{\mathrm{p}}$. If this ratio is $<1$, the rings are inferred to be the result of dust traps. With this ratio a plausible range of widths for gas pressure bumps $w_{\text {gas }}$ at the radial location of the dust rings can also be determined, in turn yielding a range of values for the ratio of the viscosity parameter to the local Stokes number (Dullemond et al. 2018, Equation 21),

$\frac{\alpha_{\text {turb }}}{\mathrm{St}}=\left[\left(\frac{w_{\text {gas }}}{w_{\text {dust }}}\right)^{2}-1\right]^{-1}$. 

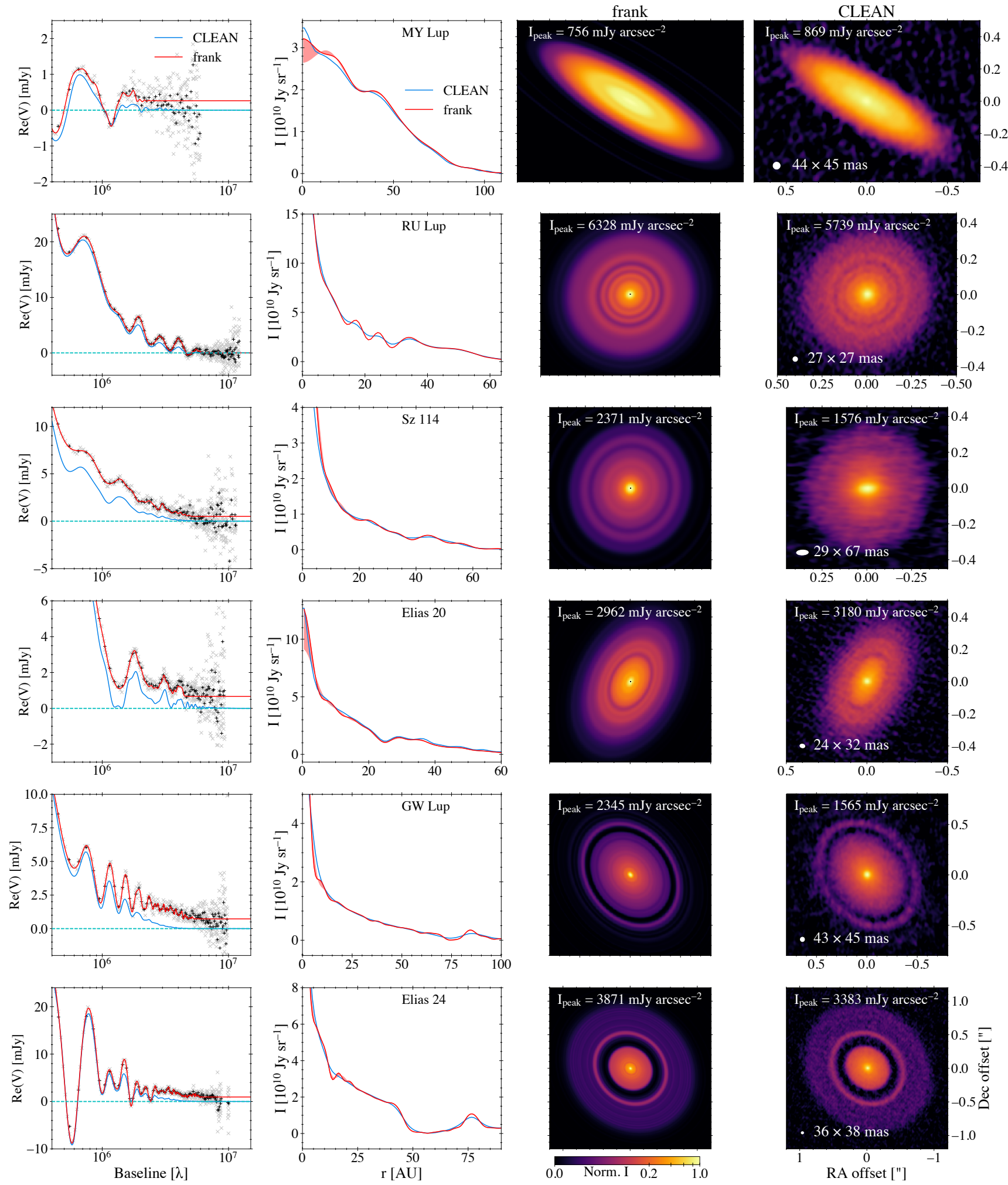

Figure 6. Substructure in extended discs As in Fig. 5, but for the extended ( $>50$ au) DSHARP discs in Sec. 5.2. 

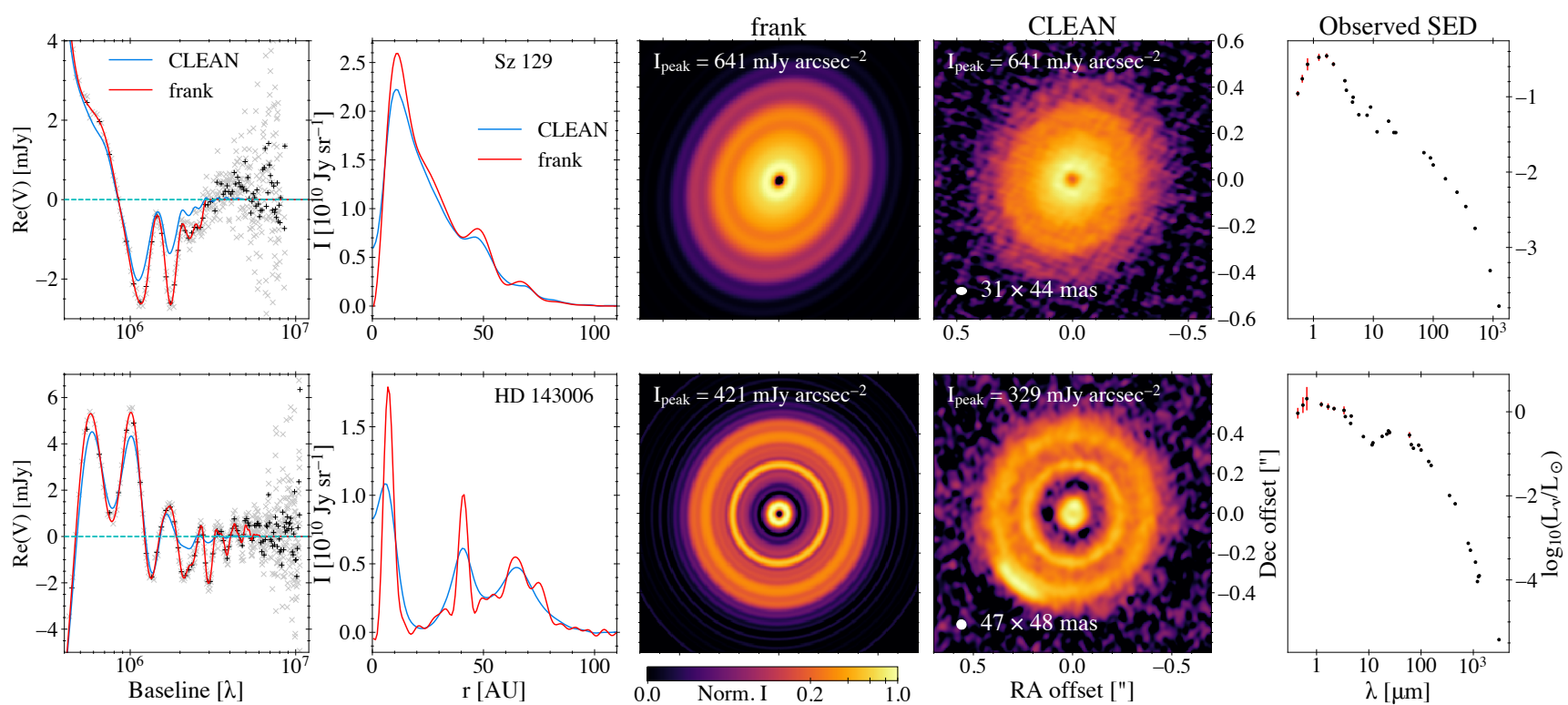

Figure 7. Evidence for inner cavities

As in Fig. 5, but for the DSHARP discs showing indications of inner cavities. Additionally shown are the observed spectral energy distributions (Andrews et al. 2018). The azimuthally localized bright arc along the outer edge of the outer ring in the CLEAN image for HD 143006 is erroneously visualized as a symmetric feature in the frank image (because the model is 1D) and manifests in the frank brightness profiles as the 'bump' at 77 au.

The lower this ratio, the lower the threshold to induce the streaming instability. Rosotti et al. 2020 take a similar approach, using the dust ring widths together with deviations from Keplerian velocity inferred from the ${ }^{12} \mathrm{CO}$ observations in AS 209 and HD 163296 to measure $\alpha_{\text {turb }} /$ St. According to their Equation 1,

$\frac{\alpha_{\text {turb }}}{\mathrm{St}}=-\frac{2 w_{\mathrm{dust}}^{2}}{r_{0}} \frac{v_{\mathrm{k}}^{2}}{c_{\mathrm{S}}^{2}} \frac{\mathrm{d}}{\mathrm{dr}}\left(\frac{\delta v_{\phi}}{v_{\mathrm{k}}}\right)$.

Here $r_{0}$ is the radial location of the dust ring, $v_{\mathrm{k}}$ the local Keplerian velocity, $c_{\mathrm{S}}$ the sound speed, and $\delta v_{\phi}=v_{\phi}-v_{\mathrm{K}}$ is the deviation from Keplerian.

Following the procedure in Dullemond et al. (2018) to determine dust ring widths, we find each of the 8 rings in the frank profiles are narrower than even the deconvolved widths in Dullemond et al. (2018), by a mean $24 \%$. The frank widths are also narrower than the 4 of these rings examined in Rosotti et al. (2020) by a mean $13 \%$. Table 4 compares the frank widths to those in Dullemond et al. (2018) and Rosotti et al. (2020), as well as the corresponding estimates of $w_{\text {dust }} / h_{\mathrm{p}}$. The narrower frank dust rings yield a reduction in estimates of $\alpha_{\text {turb }} /$ St by a mean $47 \%$ relative to Dullemond et al. 2018 (for $w_{\min }$, the minimum width of the gas pressure bump; see that work) and by a mean $25 \%$ relative to Rosotti et al. (2020). These results suggest the dust ring widths in Dullemond et al. (2018) and Rosotti et al. (2020) are overestimates, and that smaller values of $\alpha_{\text {turb }}$ (or larger values of $\mathrm{St}$ ) are thus needed to agree with the true (unknown) ring widths. A smaller ratio of $\alpha_{\text {turb }} /$ St would in turn correspond to a lower threshold for inducing the streaming instability.

To emphasize the importance of an accurate visibility fit, we note that Dullemond et al. (2018) find the deconvolved ring widths are in some cases wider, but in others narrower, than the widths determined by parametrically fitting the visibilities for AS 209 (Guzmán et al. 2018b), HD 163296 (Isella et al. 2018) and HD 143006 (Pérez et al. 2018; see Appendix C in Dullemond et al. 2018). The frank profiles instead yield narrower rings than the deconvolved widths in Dullemond et al. 2018 in all cases, because frank is fitting structure in the observed visibilities to longer baseline than the parametric visibility fits. Comparing the frank visibility fit for HD 163296 to the parametric visibility fit in Isella et al. 2018 for example, frank accurately traces the visibilities to $\approx 3.8 \mathrm{M} \lambda$, while the parametric fit begins to show clear error beyond $\approx 0.9 \mathrm{M} \lambda$, and the frank ring widths are thus narrower.

\subsection{Spiral arms appear to extend into the spiral discs' cores}

The frank fits to the three single-disc systems in the survey exhibiting prominent spirals - WaOph 6, Elias 27 and IM Lup - show clear deviations from a smooth envelope in the discs' bright cores, which extend to $\approx 45,60$ and 30 au respectively. The imaged frank residual visibilities ${ }^{6}$ in Fig. 8 suggest these features may not be tracing symmetric gaps and rings, but instead the (azimuthally averaged) innermost components of the spiral arms. This interpretation is tentatively supported by examining polar projections of the deprojected frank imaged residuals (not shown), which appear to faintly trace the arms to moderately smaller radii than the polar plots in Huang et al. (2018b).

The model for each of these discs uses the point sourcecorrected fit (Sec. 2.1), with the profile's sensitivity to the point source visibility amplitude shown as the informal uncertainty band in Fig,. 8. The exact structure in the discs' cores should thus be taken with caution, though the features in WaOph 6 beyond $\approx 20$

\footnotetext{
6 As discussed in Sec. 2.2, an azimuthally averaged frank brightness profile is erroneous for any radius at which the brightness is not symmetric. However because frank correctly fits for the averaged brightness in each annulus, subtracting the fit from the observed visibilities effectively isolates asymmetric structure in a residual image (analogous to the same procedure with CLEAN fits in Figure 1 of Huang et al. 2018b).
} 
Table 4. Dust trapping constraints from frank rings (see Sec. 5.3.1). Column (1): Disc name. (2): Ring name in Huang et al. (2018a). (3): Measured frank dust

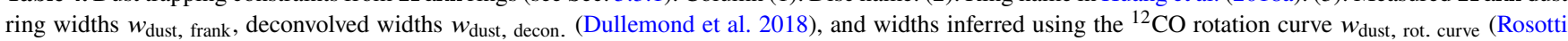
et al. 2020). (4): Ratio of the ring widths in (3) to the pressure scale height $h_{\mathrm{p}}$. (5): Ratio of turbulent viscosity to Stokes number $\alpha_{\text {turb }} /$ St, using minimum gas

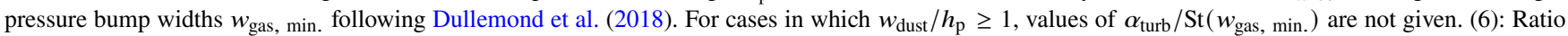
of turbulent viscosity to Stokes number $\alpha_{\text {turb }} /$ St, using gas pressure bump widths $w_{\text {gas, }}$ rot. curve following Rosotti et al. (2020). Widths $w$ in columns (3) - (6) are defined as the standard deviation of a Gaussian.

\begin{tabular}{|c|c|c|c|c|c|}
\hline Disc & $\begin{array}{c}\text { Ring } \\
\text { identifier } \\
\text { (2) }\end{array}$ & $\begin{array}{c}w_{\text {dust, frank }}[\mathrm{au}] \\
\left(w_{\text {dust, decon. }}[\mathrm{au}]\right) \\
\left\{w_{\text {dust, rot. curve }}[\mathrm{au}]\right\} \\
(3)\end{array}$ & $\begin{array}{c}w_{\text {dust, frank }} / h_{\mathrm{p}} \\
\left(w_{\text {dust, decon. }} / h_{\mathrm{p}}\right) \\
\left\{w_{\text {dust, rot. curve }} / h_{\mathrm{p}}\right\} \\
(4)\end{array}$ & $\begin{array}{c}\alpha_{\text {turb }} / \operatorname{St}\left(w_{\text {gas, min. }}, \text { frank }\right. \\
\left(\alpha_{\text {turb }} / \operatorname{St}\left(w_{\text {gas, min., decon. }}\right)\right) \\
(5)\end{array}$ & $\begin{array}{c}\alpha_{\text {turb }} / \operatorname{St}\left(w_{\text {gas, }} \text { rot. curve, frank }\right) \\
\left\{\alpha_{\text {turb }} / \operatorname{St}\left(w_{\text {gas, rot. curve }}\right)\right\} \\
(6) \\
\end{array}$ \\
\hline AS 209 & B74 & $2.86(3.38)\{3.39 \pm 0.06\}$ & $0.5(0.6)\{0.6\}$ & $0.35(0.57)$ & $0.13\{0.18 \pm 0.04\}$ \\
\hline AS 209 & B120 & $3.63(4.11)\{4.12 \pm 0.07\}$ & $0.4(0.4)\{0.4\}$ & $0.14(0.19)$ & $0.10\{0.13 \pm 0.02\}$ \\
\hline Elias 24 & B77 & $3.41(4.57)$ & $0.5(0.6)$ & $0.29(0.66)$ & \\
\hline HD 163296 & B67 & $6.32(6.84)\{6.85 \pm 0.03\}$ & $1.5(1.6)\{1.6\}$ & $-(-)$ & $0.19\{0.23 \pm 0.03\}$ \\
\hline HD 163296 & B100 & $3.80(4.67)\{4.66 \pm 0.08\}$ & $0.5(0.7)\{0.7\}$ & $0.40(0.77)$ & $0.03\{0.04 \pm 0.01\}$ \\
\hline GW Lup & B85 & $3.12(4.80)$ & $0.4(0.6)$ & $0.21(0.68)$ & \\
\hline HD 143006 & B41 & $2.09(3.90)$ & $1.0(1.9)$ & $-(-)$ & \\
\hline HD 143006 & B65 & $4.99(7.31)$ & $1.4(2.0)$ & $-(-)$ & \\
\hline
\end{tabular}

au, in Elias 27 beyond $\approx 15 \mathrm{au}$, and throughout the inner disc in IM Lup are robust to this informal uncertainty.

\subsection{The most structured DSHARP sources have morphologically similar inner discs}

frank fits to the three most highly structured DSHARP discs HD 163296, AS 209 and HD 142666 - in Fig. 9 more fully resolve gaps and rings present in the CLEAN profiles, especially the gap-ring pair in each source interior to $15 \mathrm{au}$ (noted in Table 3). The frank profiles also show new substructure in the inner disc of each source that is strikingly similar: a gap-ring pair, immediately exterior to which is a gap that shows a brightness excess (potentially a pressure bump) on both of its wings, and exterior to this a shallow depression (this region is highlighted for each source in Fig. 9). Whether this morphological similarity, including the newly identified features, is due to the same physical process, e.g., an embedded planet, would require detailed hydrodynamic simulations that are beyond the scope of this work.

\subsection{Deep gap morphologies in frank profiles potentially indicate embedded planets}

The frank brightness profiles for the six DSHARP discs shown in Fig. 10 - GW Lup, Elias 24, HD 163296, AS 209, SR 4, and HD 143006 - show that deep gaps which were already prominent in the CLEAN profiles become deeper and/or wider with sharper edges, as well as more structured in some cases. The detailed structure within the gaps in the frank profiles varies weakly as the fit's SNR criterion is varied (recall that we have accepted some low amplitude, short spatial period noise in the profiles as a tradeoff for fitting the visibilities out to baselines at which the binned data SNR approaches unity). Insensitive to the exact fit is the presence of local maxima exterior to the gaps, as well as less prominent maxima or shallow slopes interior to the gaps. Some of the gap morphologies (both the structure within the gap and on its edges) are qualitatively similar to the dust surface density distribution surrounding a gapopening planet in hydrodynamic simulations (particularly those for a stationary or slowly migrating planet in Meru et al. 2018 and Nazari et al. 2019). However detailed simulations would be required to confirm agreement in any individual case; we leave this to a future work. The four gaps shaded in gray in Fig. 10 have a claimed planet detection: in GW Lup (Pinte et al. 2020), Elias 24 (Jorquera et al.
2020) and both gaps in HD 163296 (Teague et al. 2018; Pinte et al. 2018); the gaps shaded in pink do not have a detection.

\subsection{A geometric viewing effect traces disc vertical structure}

Ten of the 20 DSHARP sources (noted in Table 2) have frank residual visibilities that when imaged exhibit a clear two-fold brightness asymmetry in the inner disc, oriented about the disc's major axis. The imaged frank residuals for these sources are shown in Fig. B1. Fig. 11 demonstrates the most prominent case, Elias 24, in which the asymmetry spans the entirety of the inner disc. This brightness asymmetry across the inner disc can be explained by a geometric viewing effect, provided the disc is optically thick, has finite thickness, and is not viewed exactly face-on. In such a case the observer sees the disc photosphere like the inclined interior of a bowl, where the angle between the local surface normal and the line of sight to the observer varies with azimuth. Since the maximum brightness is seen on the side of the disc surface that is more angled towards the observer (i.e., on the far side of the major axis), the brightness asymmetry can be used to trace the inner disc vertical structure.

This interpretation is supported by considering that among the subsample of 10 discs in which we see the asymmetry in the frank imaged residuals, a corresponding asymmetry was identified in the CLEAN images or their residuals for six sources: in the inner $5-10$ au of HD 142666, HD 163296 and Sz 129 (Huang et al. 2018a); and in the core of the survey's three discs with spiral structure, Elias 27, IM Lup and WaOph 6 (Huang et al. 2018b). The $12 \mathrm{CO}$ $J=2-1$ emission indicates the brighter region is on the disc's far side in all six cases (Huang et al. 2018a; Isella et al. 2018), consistent with our geometric interpretation. Huang et al. (2018a) posit the brightness asymmetry in HD 142666, HD 163296 and Sz 129 could be attributed to viewing the interior surface of a finite thickness ring, while we additionally see the asymmetry in sources such as Elias 24, where it spans the entirety of the (fairly smooth) inner disc. Huang et al. (2018b) attribute the brightness asymmetries in the spiral discs to an imperfect determination of the disc phase center, though they note that asymmetric brightness may also be caused by vertical structure.

Additionally, the 10 discs in which we see the brightness asymmetry all have a $1.25 \mathrm{~mm}$ optical depth as calculated in Huang et al. (2018a) that is $\approx 1$ in the inner disc (and if the brightness asymmetry is tracing vertical structure, the true optical depth may be $\gg 1$ ). Placing quantitative constraints on vertical scale height and 

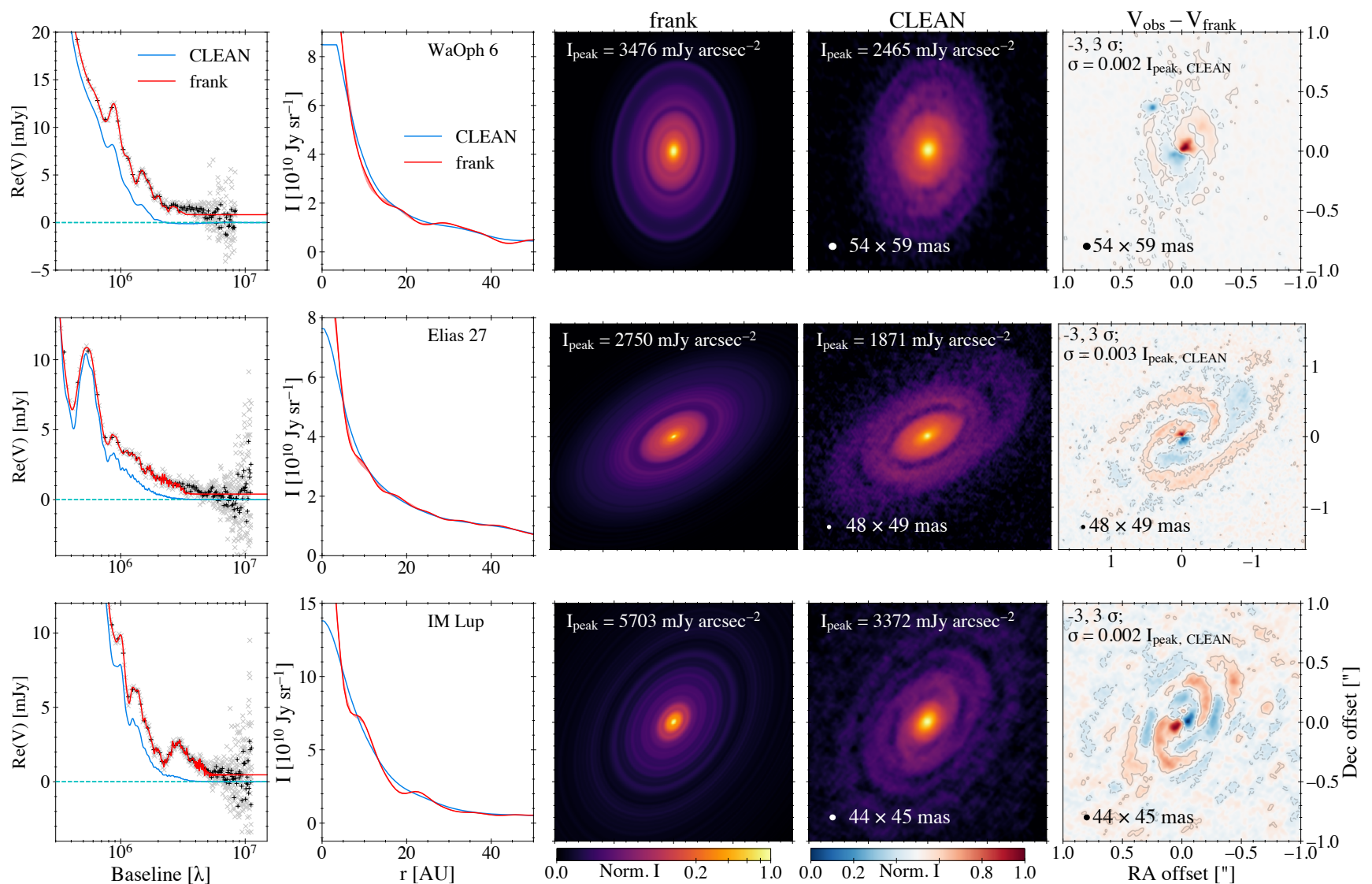

Figure 8. Tracing spiral arms into their disc's cores

As in Fig. 5, but for the DSHARP discs exhibiting strong spiral structure. The visibility plots here zoom on baselines $>0.30 \mathrm{M} \lambda$ (corresponding to spatial scales $<0.69^{\prime \prime}$ ). Additionally shown are the frank residual visibilities imaged (0 CLEAN iterations). Residual images use a linear color scale (a normalized color bar is shown, and the $\sigma$ value for each image is given). Azimuthal asymmetries in CLEAN images are erroneously visualized as symmetric features in the frank images because the frank model is 1D.

optical depth using the brightness asymmetry will be addressed in a future work. Investigating potential alternative origins of the observed brightness asymmetry in Appendix B, we find that a simple warp (inclination misalignment between an inner and outer disc) does not yield an asymmetric brightness pattern oriented about the major axis, and an incorrect source phase center does not explain the presence of this asymmetry across so many of the DSHARP sources.

\section{CONCLUSIONS}

Finding the effective resolution of CLEAN images in the DSHARP survey corresponds to an increase in the CLEAN beam width by an average factor of 1.16 , we used frank to accurately fit the 1D visibility distribution for each of the 20 DSHARP sources to a mean factor of 4.3 longer baseline than brightness profiles extracted from the CLEAN images and a factor of 3.0 longer baseline than the CLEAN models. This yielded super-resolution brightness profiles for each source that more highly resolved azimuthally symmetric (and asymmetric) disc substructure seen in the CLEAN images. The frank fits additionally identified new features - an extra gap in the inner 20 au of SR 4 and Elias 24, as well as new pressure bumps and depressions in the inner 30 au of HD 142666, HD 163296 and AS 209. Overall the analysis demonstrated two key points: the
DSHARP sources - already found to ubiquitously contain gaps and rings in Huang et al. (2018a) - are even more densely structured, especially interior to $30 \mathrm{au}$; and the gaps and rings detected in the CLEAN images, despite in many cases having widths $2-3 \times$ that of the CLEAN beam, become deeper and wider (gaps) or narrower and brighter (rings) when we fit the data with a technique not subject to CLEAN beam convolution.

We further identified new trends in substructure across the survey:

- substructure in compact discs: frank profiles for all three compact $\left(R_{\max }<50 \mathrm{au}\right)$, single-disc systems showed substructure, suggesting it may be frequent in compact sources

- substructure in extended discs: frank profiles for six extended $\left(R_{\max }>50 \mathrm{au}\right.$ ), fairly smooth DSHARP sources found indications of a change in slope in the innermost disc, implying the interior regions of discs may commonly be structured

- potential transition discs: frank profiles for two of the oldest discs in the sample suggested they have cleared inner cavities, which may indicate they are dispersing

- spiral arms in disc cores: frank profiles for the three singledisc systems with prominent spirals suggested the spiral arms reach into the discs' cores

- inner disc morphologies: frank profiles for the three most structured DSHARP discs exhibited highly similar substructure 

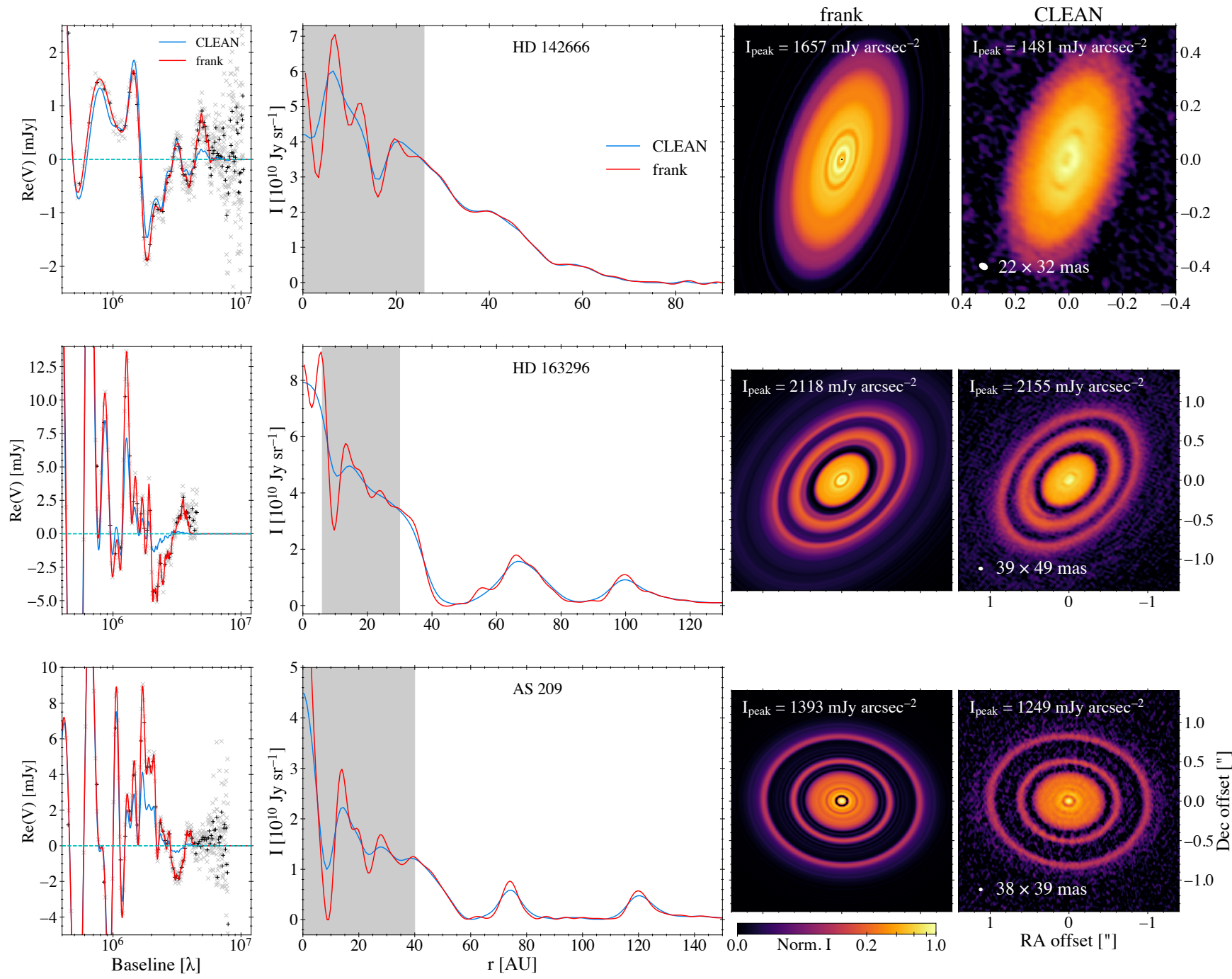

Figure 9. Highly structured discs

As in Fig. 5, but for the DSHARP discs exhibiting the highest density of substructures. The azimuthally localized bright arc along the inner edge of the intermediate ring in the CLEAN image for HD 163296 is erroneously visualized as a symmetric feature in the frank image (because the model is 1D) and manifests in the frank brightness profile as the 'bump' at 55 au. The shaded regions show morphological similarities across discs as discussed in Sec. 5.5.

morphology in their inner $40 \mathrm{au}$, indicating the same physical processes, e.g., the presence of a companion, may be responsible

- gap morphologies: frank profiles for six survey discs that already had prominent gaps in the CLEAN images showed these features to have greater depth and/or more structure (both within the gap and on its wings)

We found that lower values of $\alpha_{\text {turb }} /$ St than determined in Dullemond et al. (2018) and Rosotti et al. (2020) are needed to explain the super-resolved ring widths in AS 209, Elias 24, HD 163296, GW Lup, and HD 143006. Finally, the frank fits also found clear evidence of a geometric viewing effect in 10 of the 20 DSHARP sources that traces inner disc vertical structure.

The extent to which these substructure trends are present in surveys and individual datasets with different biases (DSHARP consists primarily of bright, large discs; Andrews et al. 2018) is a question we will address in subsequent work. Those trends that do hold beyond DSHARP may offer the potential to broadly in- form open questions on the physical mechanisms underlying dust substructure in protoplanetary discs.

On the technical side, the analysis in this work demonstrated that frank, and super-resolution fitting techniques more generally, can consistently extract more 1D substructure information from submm disc observations than both CLEAN images and CLEAN models. There is a clear limitation with frank in that it reconstructs the $1 \mathrm{D}$ brightness of a source, rather than the $2 \mathrm{D}$ brightness as in a CLEAN image. However, for the purpose of obtaining a 1D brightness profile of a source (under the assumptions of axisymmetry and known source geometry), frank will yield a more accurate (higher resolution) result, without a loss in sensitivity, compared to extracting an azimuthally averaged profile from the CLEAN image. Super-resolution techniques can provide new insights from existing datasets, better informing physical inference without requiring deeper and/or longer baseline observations. In practice these tools can also be approachable and efficient; performing a frank fit requires nontrivial choices for only two hyperparameters (the 

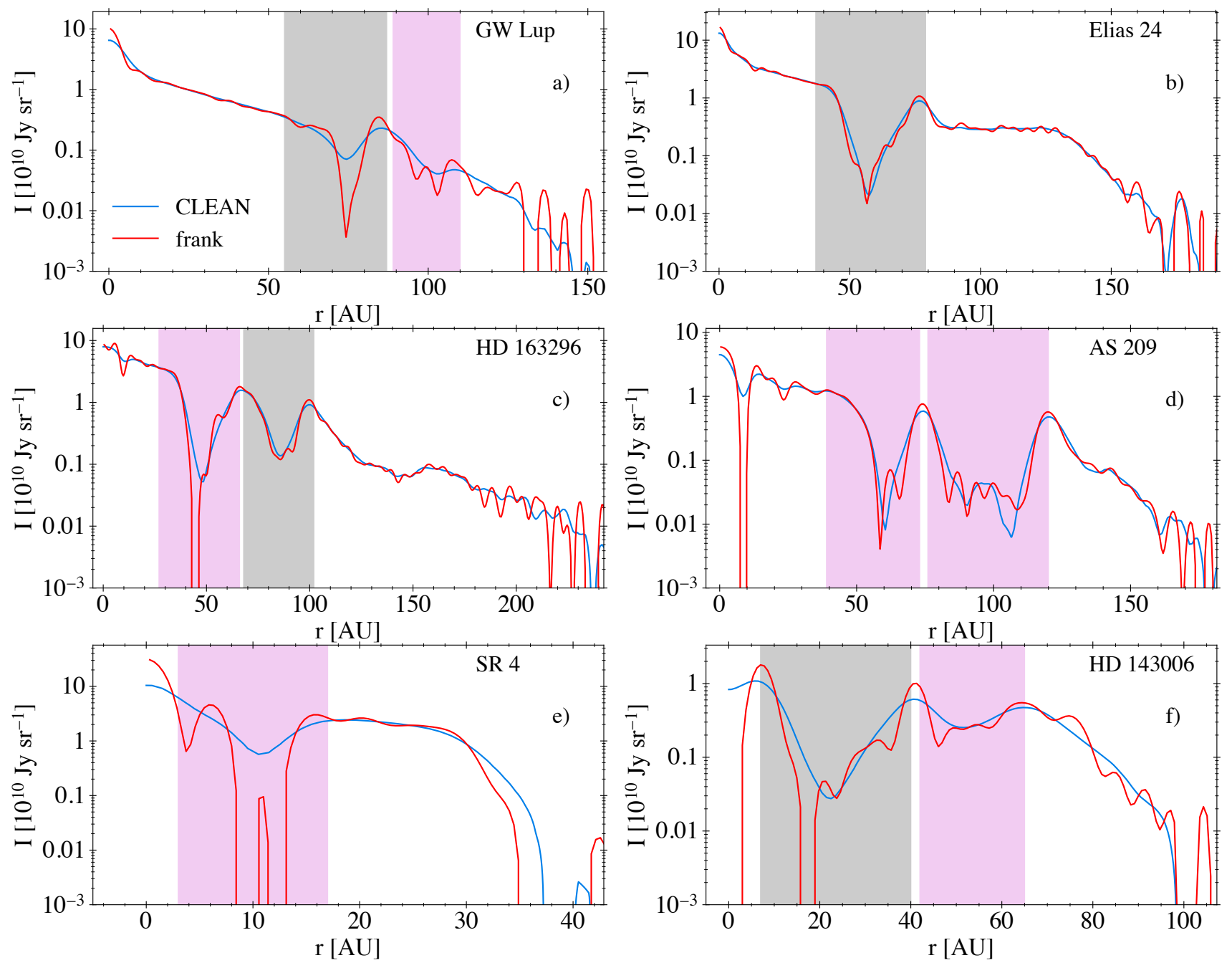

Figure 10. Morphologies for deep and structured gaps

frank and CLEAN brightness profiles in logarithmic brightness for DSHARP discs whose frank profiles have gaps that are either appreciably deeper or contain more structure than seen in the CLEAN profiles. Gap regions are shaded for identification; those shaded in gray have a claimed planetary detection (either from gas kinematics or direct imaging), and those in pink have no detection.

parameter space for each being small), and the frank fits shown in this work all took $\lesssim 1$ min to run. frank is open source code, available at https://github.com/discsim/frank and documented at https://discsim.github.io/frank. All frank fits in this work are available at https://zenodo.org/record/5587841.

\section{ACKNOWLEDGEMENTS}

JJ thanks H. Vaivao for his comments on the work. This work was supported by the STFC consolidated grant ST/S000623/1. G.R. acknowledges support from the Netherlands Organisation for Scientific Research (NWO, program number 016.Veni.192.233) and from an STFC Ernest Rutherford Fellowship (grant number ST/T003855/1). This work has also been supported by the European Union's Horizon 2020 research and innovation programme under the Marie Sklodowska-Curie grant agreement No. 823823 (DUSTBUSTERS).
Software: NumPy (Walt et al. 2011), SciPy (Virtanen et al. 2019), Matplotlib (Hunter 2007), Astropy (Astropy Collaboration et al. 2013, 2018), Jupyter Notebook (Kluyver et al. 2016), CASA (McMullin et al. 2007), uvplot (Tazzari 2017)

\section{DATA AVAILABILITY}

The data underlying this article are available in the DSHARP Data Release at https://bulk.cv.nrao.edu/almadata/lp/DSHARP. The datasets were derived from this previously released source in the public domain.

\section{REFERENCES}

ALMA Partnership T., et al., 2015, The Astrophysical Journal Letters, 808, L3

Akiyama K., et al., 2017, AJ, 153, 159 

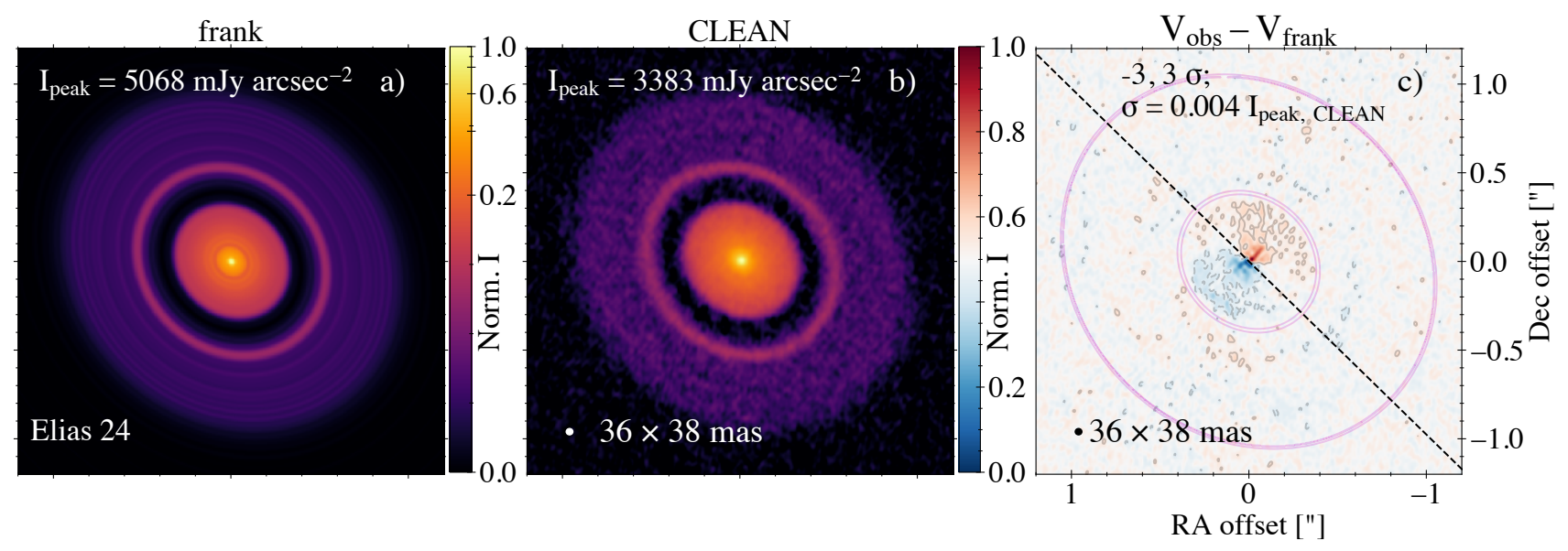

Figure 11. A geometric viewing effect tracing disc vertical structure

a) For Elias 24 , an image of the frank profile swept over $2 \pi$ and reprojected.

b) The CLEAN image. The frank and CLEAN images of each disc use the same $\operatorname{arcsinh} \operatorname{stretch}\left(I_{\text {stretch }}=\operatorname{arcsinh}(I / a) / \operatorname{arcsinh}(1 / a), a=0.02\right)$, but different brightness normalization. The generic color bar gives the normalized color scale, and the peak brightness is listed on both images.

c) The frank residual visibilities imaged (0 CLEAN iterations), with contours overplotted, as well as additional lines tracing the outer edge of the inner disc and the disc outer edge (from (a)), and a dashed line along the fitted position angle (as a proxy for the disc's major axis). The residual image is convolved with the published CLEAN beam and uses a linear color scale. The shown $3 \sigma$ contours correspond to a residual brightness $<1 \%$ of the local average brightness in the CLEAN image at the outer edge of the inner disc, 42 au. The residual image uses a linear color scale (a normalized color bar is shown, and the $\sigma$ value for each image is given).

Andrews S. M., et al., 2016, The Astrophysical Journal, 820, L40

Andrews S. M., et al., 2018, ApJ, 869, L41

Astropy Collaboration et al., 2013, A\&A, 558, A33

Astropy Collaboration et al., 2018, aj, 156, 123

Baddour N., Chouinard U., 2015, Journal of the Optical Society of America A, 32, 611

Casassus S., Cabrera G. F., Förster F., Pearson T. J., Readhead A. C. S., Dickinson C., 2006, ApJ, 639, 951

Casassus S., et al., 2013, Nature, 493, 191

Chael A. A., Johnson M. D., Narayan R., Doeleman S. S., Wardle J. F. C., Bouman K. L., 2016, ApJ, 829, 11

Clark B. G., 1980, A\&A, 89, 377

Clarke C. J., et al., 2018, ApJ, 866, L6

Cornwell T. J., 2008, IEEE Journal of Selected Topics in Signal Processing, 2,793

Czekala I., Zawadzki B., Loomis R., Grzybowski H., Frazier R., Quinn T., 2021, MPoL-dev/MPoL: v0.1.1 Release, doi:10.5281/zenodo.4939048, https://doi.org/10.5281/zenodo. 4939048

Dullemond C. P., et al., 2018, ApJ, 869, L46

Espaillat C., et al., 2014, in Beuther H., Klessen R. S., Dullemond C. P., Henning T., eds, Protostars and Planets VI. p. 497 (arXiv : 1402 .7103), doi:10.2458/azu_uapress_9780816531240-ch022

Event Horizon Telescope Collaboration et al., 2019, ApJ, 875, L4

Gull S. F., Daniell G. J., 1978, Nature, 272, 686

Guzmán V. V., et al., 2018a, ApJ, 869, L48

Guzmán V. V., et al., 2018b, ApJ, 869, L48

Högbom J. A., 1974, A\&AS, 15, 417

Honma M., Akiyama K., Uemura M., Ikeda S., 2014, Publications of the Astronomical Society of Japan, 66

Huang J., et al., 2018a, ApJ, 869, L42

Huang J., et al., 2018b, ApJ, 869, L43

Hunter J. D., 2007, Computing in Science \& Engineering, 9, 90

Isella A., et al., 2018, ApJ, 869, L49

Jennings J., Booth R. A., Tazzari M., Rosotti G. P., Clarke C. J., 2020, MNRAS, 495, 3209

Jorquera S., et al., 2020, arXiv e-prints, p. arXiv:2012.10464

Keppler M., et al., 2019, Astronomy \& Astrophysics, 625, A118

Kluyver T., et al., 2016, in Loizides F., Schmidt B., eds, Positioning and
Power in Academic Publishing: Players, Agents and Agendas. pp 87 90

Kudo T., Hashimoto J., Muto T., Liu H. B., Dong R., Hasegawa Y., Tsukagoshi T., Konishi M., 2018, ApJ, 868, L5

Kuramochi K., Akiyama K., Ikeda S., Tazaki F., Fish V. L., Pu H.-Y., Asada K., Honma M., 2018, ApJ, 858, 56

Kurtovic N. T., et al., 2018, ApJ, 869, L44

McMullin J. P., Waters B., Schiebel D., Young W., Golap K., 2007, CASA Architecture and Applications. p. 127

Meru F., Rosotti G. P., Booth R. A., Nazari P., Clarke C. J., 2018, Monthly Notices of the Royal Astronomical Society, 482, 3678

Nakazato T., Ikeda S., Akiyama K., Kosugi G., Yamaguchi M., Honma M., 2019, in Teuben P. J., Pound M. W., Thomas B. A., Warner E. M., eds, Astronomical Society of the Pacific Conference Series Vol. 523, Astronomical Data Analysis Software and Systems XXVII. p. 143

Narayan R., Nityananda R., 1986, ARA\&A, 24, 127

Nazari P., Booth R. A., Clarke C. J., Rosotti G. P., Tazzari M., Juhasz A., Meru F., 2019, MNRAS, 485, 5914

Oppermann N., Selig M., Bell M. R., Enßlin T. A., 2013, Phys. Rev. E, 87, 032136

Pérez L. M., et al., 2018, ApJ, 869, L50

Pérez S., Casassus S., Baruteau C., Dong R., Hales A., Cieza L., 2019, arXiv e-prints,

Perkins S., Marais P., Zwart J., Natarajan I., Tasse C., Smirnov O., 2015, Astronomy and Computing, 12, 73

Pinte C., et al., 2018, The Astrophysical Journal, 860, L13

Pinte C., et al., 2019, Nature Astronomy, p. 419

Pinte C., et al., 2020, ApJ, 890, L9

Remijan A., et al., 2019, ALMA Doc. 7.3, ver. 1.0

Rosotti G. P., Teague R., Dullemond C., Booth R. A., Clarke C. J., 2020, MNRAS, 495, 173

Sutton E. C., Wandelt B. D., 2006, ApJS, 162, 401

Tazzari M., 2017, mtazzari/uvplot, doi:10.5281/zenodo.1003113, https: //doi.org/10.5281/zenodo. 1003113

Tazzari M., Beaujean F., Testi L., 2018, MNRAS, 476, 4527

Teague R., Bae J., Bergin E. A., Birnstiel T., Foreman-Mackey D., 2018, ApJ, 860, L12

Virtanen P., et al., 2019, SciPy 1.0-Fundamental Algorithms for Scientific 
Computing in Python (arXiv: 1907.10121)

Walt S. v. d., Colbert S. C., Varoquaux G., 2011, Computing in Science \& Engineering, 13, 22

Yamaguchi M., et al., 2020, The Astrophysical Journal, 895, 84 source offset fit is real; this ambiguity motivates our treatment of the difference between these two fits as an informal uncertainty estimate in all discs where we use the point source-corrected model in the main text.

\section{APPENDIX A: POINT SOURCE-CORRECTED FITS}

To demonstrate the effect of a point source-corrected fit, Fig. A1(a) - (b) compares a model generated with this approach to two standard frank fits for GW Lup. In panel (b), the observed visibilities remain systematically positive at the longest baselines, i.e., do not converge on zero. Their offset is $0.7 \mathrm{mJy}$; for reference, $\operatorname{Re}(\mathrm{V})$ plateaus at $88.9 \mathrm{mJy}$ at short baselines. First considering the two standard frank fits (which use different $\alpha$ values), the model with $\alpha=1.1$ fits the visibilities out to $\approx 7 \mathrm{M} \lambda$, at which point some of the $100 \mathrm{k} \lambda$ binned values approach zero. However because the data are noisedominated by this baseline, the corresponding brightness profile in Fig. A1(a) has noisy oscillations, most apparent at small radii. By comparison, increasing $\alpha$ to 1.3 effectively fits the data to shorter baseline, $\approx 5 \mathrm{M} \lambda$, beyond which the binned SNR start to dither about $\mathrm{SNR}=1$. The model drives toward zero (by design) once its SNR threshold is reached, which is problematic if the fit's slope at this baseline is steeper than the average slope of the true, underlying signal in the data. That appears to be the case here, as the fit's slope still translates to strong oscillations in the brightness profile in panel (a).

The point source-corrected model in Fig. A1(b) fits the data out to comparable baseline to the $\alpha=1.3$ case, but once its SNR threshold is reached, the fit takes on a constant visibility amplitude (rather than driving toward zero). This amplitude is the mean of the data beyond the baseline at which the $20 \mathrm{k} \lambda$ binned SNR first drops below unity. The strong oscillations in the innermost disc present in the standard fits are no longer apparent in the point source-corrected fit, though we do still see some small amplitude oscillations across all radii in the brightness profile, whose sensitivity we will examine below. The fit's zero slope over the data's longest baselines yields a conservative representation of features on the corresponding spatial scales in the brightness profile, which we prefer because of the ambiguity in where the true visibility signal converges on zero.

While for practical purposes the point source-corrected model is the best approach we have at present to fit a visibility distribution that does not clearly converge on zero, it has limitations. First, because it involves fitting frank to a visibility distribution from which we have subtracted a constant offset, the SNR of the resulting data are not identical to those of the observed data. This is why the point source-corrected model in Fig. A1(b) fits the visibilities beyond $\approx 4 \mathrm{M} \lambda$ less closely than the shown standard fits, despite using a lower $\alpha$.

Second, while we have determined the point source amplitude by taking the mean of the longest baseline visibilities, they are in general dominated by noise and so not necessarily an accurate indication of the true signal. We thus test how the applied point source offset affects the frank visibility fit and in turn substructure in the brightness profile. Fig. A1(d) shows the visibility fit for GW Lup when we increase the point source offset to $1.5 \times$ the mean of the long baseline data. This offset expectedly yields larger amplitude (negative) residuals in panel (e), while also reducing structure in the brightness profile interior to $\approx 0.1^{\prime \prime}$ in panel $(\mathrm{c})$. The reduced prominence of structure seems less correct than the fit with a lower point source offset based on the residuals in (e). However it is also not clear that the structure interior to $0.1^{\prime \prime}$ in the smaller point

\section{APPENDIX B: RESIDUAL IMAGE BRIGHTNESS ASYMMETRIES}

Considering the residual brightness asymmetries in Sec. 5.7, Fig. B1 shows the frank residuals imaged for each DSHARP source. Here we present tests to determine whether the observed trend of a brightness asymmetry oriented about the major axis in 10 of the 20 sources could - instead of a geometric effect - be produced by either an incorrect source phase center or a simple warp in the form of a misalignment between the inner and outer discs (effectively an incorrect inclination). First considering a phase center error, shifting the phase center of a flat disc generates an asymmetry in the direction of the centroid error. In order to explain the observed asymmetry pattern in 10 of the 20 DSHARP discs would thus require that some aspect of fitting for the phase center (which was done by fitting a 2D Gaussian to the image) biased the error toward alignment with the disc's minor axis. We do not see how such a bias could arise.

Nevertheless, as a precaution we considered the $1 \sigma$ uncertainties in fitted right ascension and declination offsets as determined in Huang et al. (2018a), which are typically $1-3$ mas. To test whether shifting the phase center within this range could effectively erase the brightness asymmetry in the residual maps, for each DSHARP source we applied a phase center that differed from the published value by 1 or 3 mas, with the perturbation oriented along the disc's minor axis as well as at $\pi / 4$ intervals over the full $2 \pi$ in azimuth. For each of these applied phase centers, we then fit for the frank profile, and compared the resulting imaged frank residuals. Shifting the phase center in this way did change the amplitude of the brightness asymmetry in the inner disc by a factor of $\lesssim 2$, and in some cases it slightly rotated the asymmetry's orientation. But in almost all cases the asymmetry clearly persisted, suggesting it is not an artifact of an incorrect phase center.

For the 10 DSHARP discs in which we initially did not identify a clear brightness asymmetry, shifting the phase center along the disc's minor axis could in some cases create an asymmetry similar to that observed. The same was true for mock datasets in which we intentionally assigned an incorrect phase center. And 2 of these 10 sources, SR 4 and Sz 114, exhibited an asymmetry that was not aligned about the major axis; however shifting the phase center within published uncertainty $(<3$ mas) could reorient the asymmetry about the major axis. Taking all of this together, again we do not see why fitting for the phase center as described in Huang et al. (2018a) would introduce a bias along the disc's minor axis.

Next considering disc misalignment, we forward modeled mock observations emulating DSHARP datasets that have an inner disc separated from an outer ring by a deep gap. We generated images in which the inner disc's inclination was misaligned relative to the outer ring by values between $0.1-3^{\circ}$ (the published $1 \sigma$ uncertainties on inclination are $\leq 2^{\circ}$ in either direction). We then forced the geometry used to deproject the source to be that of the outer ring (separately, we also ran trials in which we fit for the geometry using a 2D Gaussian in visibility space), and fit the deprojected dataset with frank. We found that a misaligned inner disc produces a four-fold symmetric pattern oriented equivalently about the major or minor axis in the imaged frank residuals. In 

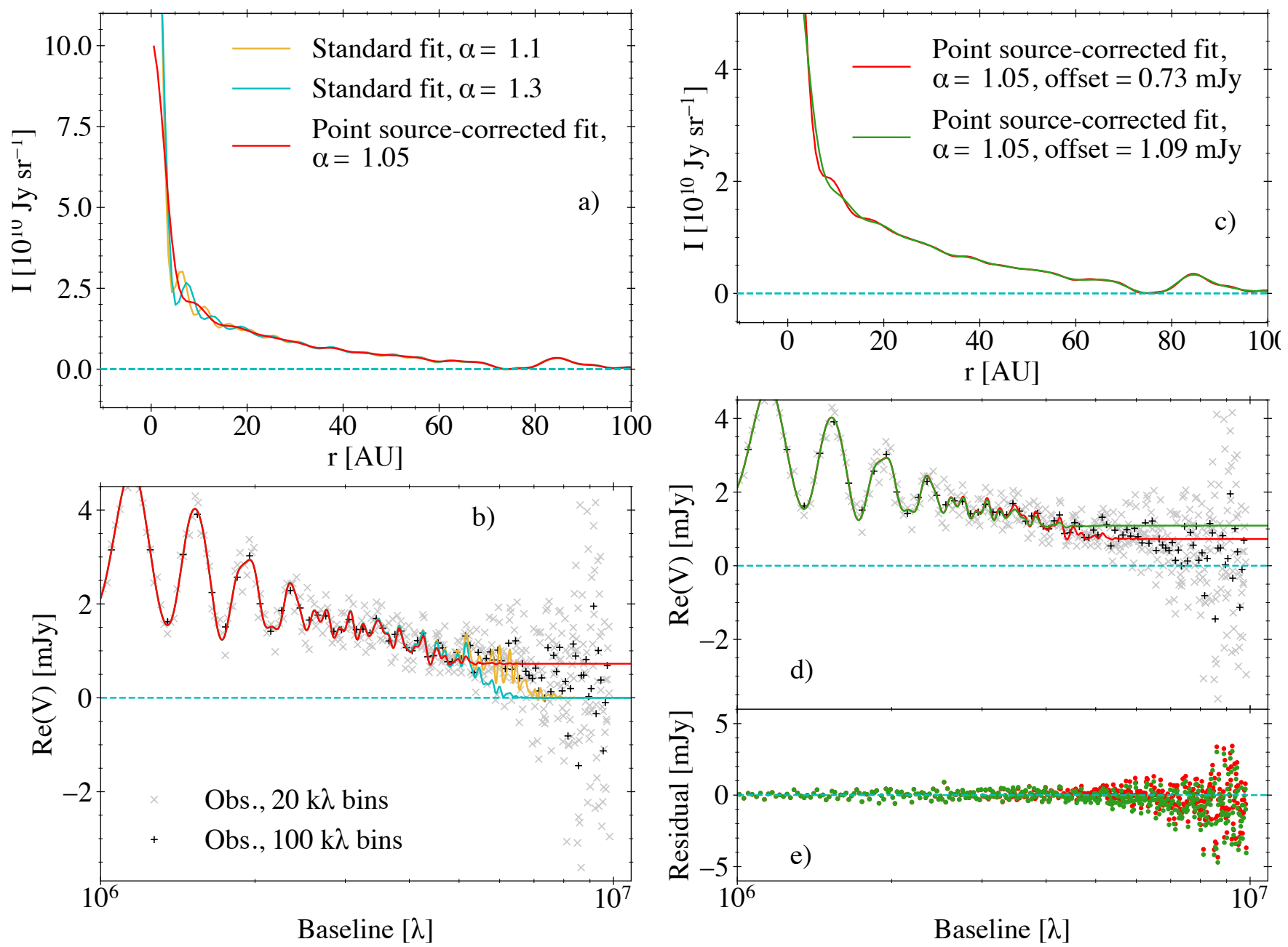

Figure A1. Effects of a point source-corrected fit

a) frank brightness profiles for two standard fits using different $\alpha$, and the profile for the point source-corrected fit shown in the main text.

b) A zoom on the data's long baselines ( $>1.0 \mathrm{M} \lambda$, corresponding to spatial scales $<0.2^{\prime \prime}$; data shown in 20 and $100 \mathrm{k} \lambda$ bins), the two standard frank fits, and the point source-corrected fit.

c) frank brightness profiles for the point source-corrected fit in (a), and a point source-corrected fit using a $1.5 \times$ larger point source amplitude.

d) As in (b), but for the two point source-corrected fits in (c).

e) Residuals (in $20 \mathrm{k} \lambda$ bins) of the two point source-corrected fits in (d).

the real observations we instead see a two-fold asymmetric pattern oriented about the major axis.

This paper has been typeset from a $\mathrm{T}_{\mathrm{E}} \mathrm{X} / \mathrm{L} \mathrm{AT} \mathrm{E} \mathrm{X}$ file prepared by the author. 

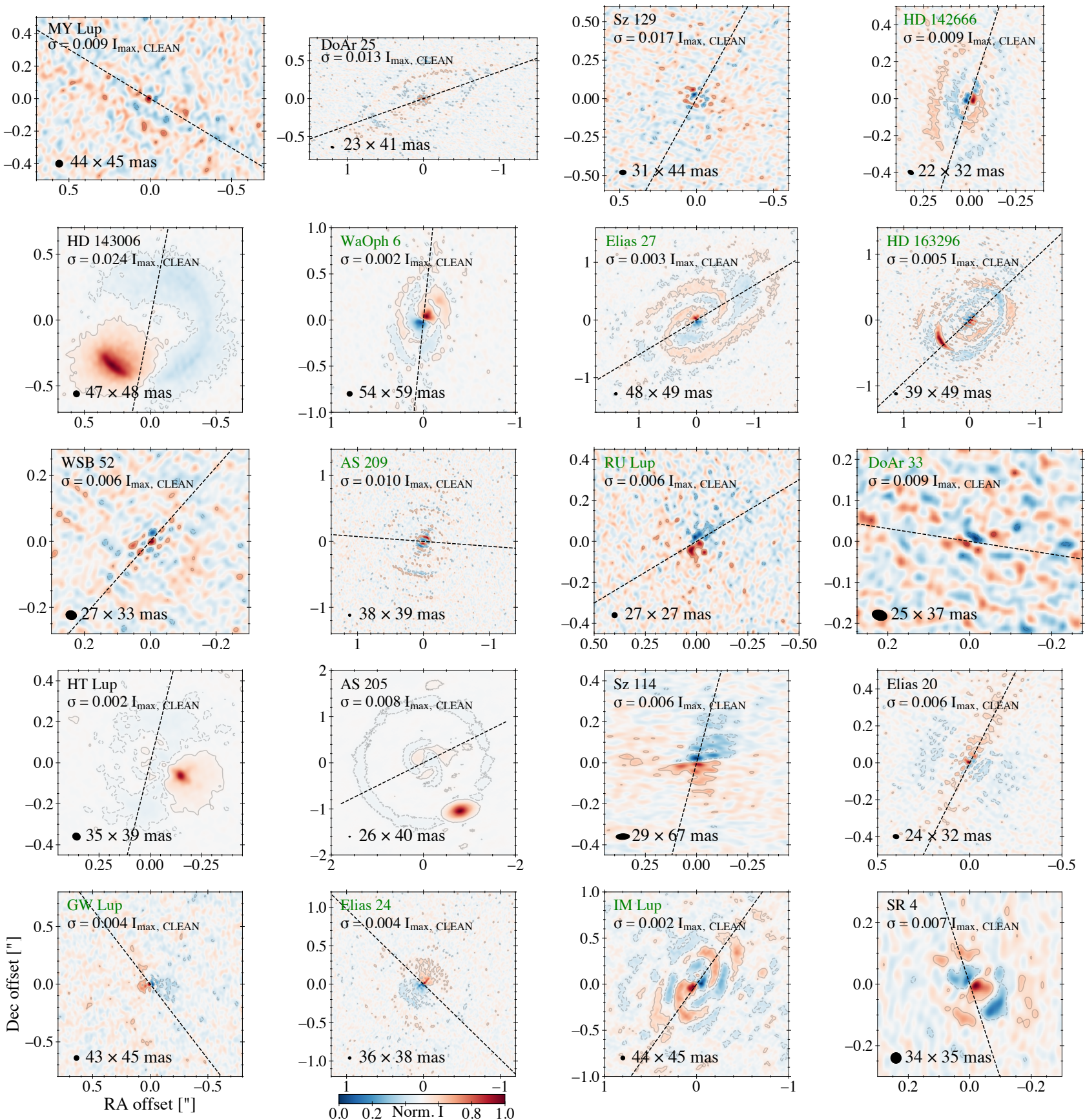

Figure B1. frank imaged residuals

The frank residual visibilities imaged ( 0 CLEAN iterations), with $\pm 3 \sigma$ contours overplotted ( $\sigma$ is given for each image), and a dashed line along the fitted position angle. The residual image is convolved with the published CLEAN beam and uses a linear color scale. Discs are ordered as in Fig. 3 . The 10 sources that exhibit a clear two-fold brightness asymmetry in the inner disc have their names shown in green. All images use a linear color scale (a normalized color bar is shown, and the $\sigma$ value for each image is given). 